\title{
Application of the Zhang-Gal-Chen Single-Doppler Velocity Retrieval to a Deep Convective Storm
}

\author{
STEVen Lazarus \\ Department of Meteorology, University of Utah, Salt Lake City, Utah \\ Alan Shapiro And Kelvin Droegemeier \\ School of Meteorology and Center for Analysis and Prediction of Storms, University of Oklahoma, \\ Norman, Oklahoma
}

(Manuscript received 24 June 1999, in final form 7 July 2000)

\section{ABSTRACT}

\begin{abstract}
The Zhang-Gal-Chen single-Doppler velocity retrieval (SDVR) technique is applied to a multicell storm observed by three radars near the Orlando, Florida, airport on 9 August 1991. This dataset is unique in that 3 min volume scans at very high spatial resolution $(200 \mathrm{~m})$ are available during a 24-min period. The retrieved (unobserved) wind, determined using only the radial wind and reflectivity from one of the radars, is compared to the (observed) winds obtained from a hybrid three-dimensional wind synthesis.

Error statistics demonstrate that the retrievals perform best when applied in a reference frame moving with the storm; however, the results also show that the specification of this frame is problematic. The findings also indicate that, in an environment where the mean flow has a critical layer, the moving reference frame is best defined as a function of height rather than a volume mean. The benefit of such a reference frame is case dependent and is best realized in regions such as a surface cold pool or upper-level divergence at storm top.

Error statistics demonstrate that the SDVR technique recovers the horizontal wind with greater accuracy than it does the vertical velocity - suggesting that for deep convection, the absence of dynamical constraints is critical. The kinematic and O'Brien techniques and a new variational technique, in which the solution to a second-order ordinary differential equation for the vertical velocity is expressed in terms of Bessel functions, are tested as possible alternatives to the SDVR vertical velocity. Results indicate that this new technique yields vertical velocities significantly better than those using the other three methods.
\end{abstract}

\section{Introduction}

The extensive coverage afforded by the national WSR-88D Doppler radar network is an important factor motivating storm-scale prediction research (Lilly 1990; Droegemeier 1990, 1997). Despite the potential for high space and time resolution data, the wind field observed by the WSR-88D is limited to the radial component, with the large distances among radars generally precluding dual-Doppler analysis. These factors, coupled with a desire to obtain a more complete representation of the storm-scale environment, have prompted research into a class of wind retrieval techniques commonly referred to as single-Doppler velocity retrieval (SDVR). Although these techniques apply different methodologies, each of them seeks to obtain the unobserved wind components by utilizing a time series of the observed

Corresponding author address: Dr. Steven Lazarus, Department of Meteorology, University of Utah, 819 William Browning Building, Salt Lake City, UT 84112.

E-mail: slazarus@met.utah.edu
single-Doppler radial velocity and/or reflectivity fields. SDVR has been applied primarily to boundary-layer phenomena such as the sea breeze and cold-air outflows, and thus its applicability to deep convection has yet to be thoroughly tested (Crook and Tuttle 1994; Shapiro et al. 1995; Sun and Crook 1994; Tuttle and Foote 1990; $\mathrm{Xu}$ et al. 1994a,b; Xu et al. 1995; Zhang and Gal-Chen 1996, hereafter ZG96).

The results of Weygandt et al. (1995), where SDVR has been applied to a deep convective-scale dataset, support the Gal-Chen hypothesis that retrievals performed in the moving reference frame are more accurate than their stationary frame counterparts (Gal-Chen 1982). The relatively large temporal rift (upward of 6 min) between successive radar scans can, even in the presence of perfect data, engender errors in estimating the reflectivity time derivative (ZG96). By defining a moving frame that minimizes the total reflectivity time derivative, the effects of aliasing can be mitigated. Additionally, if the scheme in question assumes that the velocity field is stationary, a moving reference frame is less likely to violate this assumption. It is important to 
point out, however, that there exists no unique way to define the moving reference frame, and thus the design of an "optimal" frame is likely problematic (Hane 1993).

Weygandt et al. (1995) tested two techniques for estimating storm motion: the Gal-Chen least-squares methodology (hereafter referred to as ZG), and a second method that calculates a mean horizontal velocity by minimizing departures in the observed radial velocity. Both of these methods, as applied by Weygandt et al., estimate a single volume-averaged mean wind. The latter technique delivered the best results for his particular dataset-a supercell storm. Its success may be attributable, in part, to the alignment of the principal stormscale flow with that of the mean wind at most levels. Our results indicate that in cases of near quiescent conditions, or in the presence of a critical layer, a reference frame defined by the volume-averaged mean wind may not prove to be optimal. Because we seek to obtain a frame that minimizes errors in the retrieved winds, the ZG scheme has an advantage over that of other SDVR schemes in that one can easily redefine the moving frame (e.g., from one where the mean flow is determined from a volume average to one where the mean flow is height dependent). Additionally, the ZG scheme is set up such that it can be evaluated analytically-thereby lending itself to quantifiable sensitivity analyses (Lazarus et al. 1999).

Sun and Crook (1998) applied an adjoint model to retrieve the $3 \mathrm{D}$ wind, temperature, and microphysics of a Florida airmass thunderstorm. They performed experiments using one and two radars and compared their results to aircraft measurements. They were able to capture the overall structure of the convection; however, the strength of the convection in their single-Doppler experiment was reduced over that of their dual-Doppler assimilation experiment. The Sun and Crook results are encouraging and represent a first attempt to apply adjoint methodology to retrieve wind and thermodynamic quantities for deep convection.

Most recently, Liou (1999) modified the ZG technique by introducing additional constraints (mass conservation and vorticity) to the cost function originally proposed by ZG. This modified technique was then compared with the original ZG method using simulated data. Liou shows marked improvement in the retrieved horizontal wind components using his modified method; however, the vertical wind component retrieval was less successful. Liou suggests that his technique applies best to low-elevation angle scans and does not apply it to deep convection.

These and other studies have yielded valuable information concerning the capabilities and limitations of SDVR techniques. Yet despite this work, there remains a number of unanswered questions surrounding the application of these techniques to deep precipitating systems, particularly the utility of reflectivity conservation schemes in the presence of nonconservative processes such as precipitation fallout, evaporation, and condensation, as well as the assignment of an appropriate reference frame. We attempt to address these and related issues by applying the ZG SDVR technique to a deep convective dataset. The technique is predicated on the assumptions of reflectivity conservation and velocity stationarity in the moving reference frame and uses a least squares methodology to recover the unknown wind components from volume time series of the singleDoppler radial wind and reflectivity fields.

In section 2 we discuss the dataset while in section 3 we briefly address the moving reference frame formulation. The results from a number of sensitivity tests are presented in section 4. A benchmark retrieval (based on the results obtained in section 4), and, for comparison purposes, the statistics of two other retrievals compose section 5. The retrieval of the vertical velocity, as well as alternative methods for obtaining it, is examined in section 6. A summary of the results is presented in section 7 .

\section{Dataset description}

\section{a. Technical aspects}

The three-dimensional wind field used in this work was obtained from MIT/Lincoln Lab in conjunction with the Federal Aviation Administration's Terminal Doppler Weather Radar program near the Orlando International Airport (Keohan et al. 1992). The network comprised three C-Band Doppler weather radars positioned in a triangular configuration, with baselines ranging from 16 to $20 \mathrm{~km}$ (Fig. 1). A signal-to-noise ratio of $10 \mathrm{~dB}$ was chosen as a threshold for both the reflectivity and radial velocity. Although this threshold is on the high side, the analysis was not concerned with low signal events (e.g., fine lines), it ensured that the data would be relatively noisefree (M. Wolfson 1999, personal communication). The thresholded data were first interpolated to a $200-\mathrm{m}$ Cartesian grid by averaging specified range gates wherein the radials were subdivided by azimuth, range, and height. The Cartesian-grided data were then hole-filled by both a linear and median filter with the latter being applied as a means for dealiasing. A synthetic hybrid three-dimensional wind analysis (DeLaura et al. 1989) was then applied and, within their baselines, the threedimensional wind field was recovered. DeLaura et al. applied a hybrid methodology to perform this synthesis-using both the direct (Armijo 1969) and overdetermined dual-Doppler (Ray et al. 1980) methods to obtain the three-dimensional wind field.

In addition to the hybrid wind synthesis data, singleDoppler radial velocities and reflectivity data were available from each of the three individual radars. The data are of very high temporal (3 min between volume scans) and space $(200 \mathrm{~m})$ resolution, and are available for $24 \mathrm{~min}$ (from 1923 to 1947, all times UTC). Al- 


\section{Triple-Doppler Network}

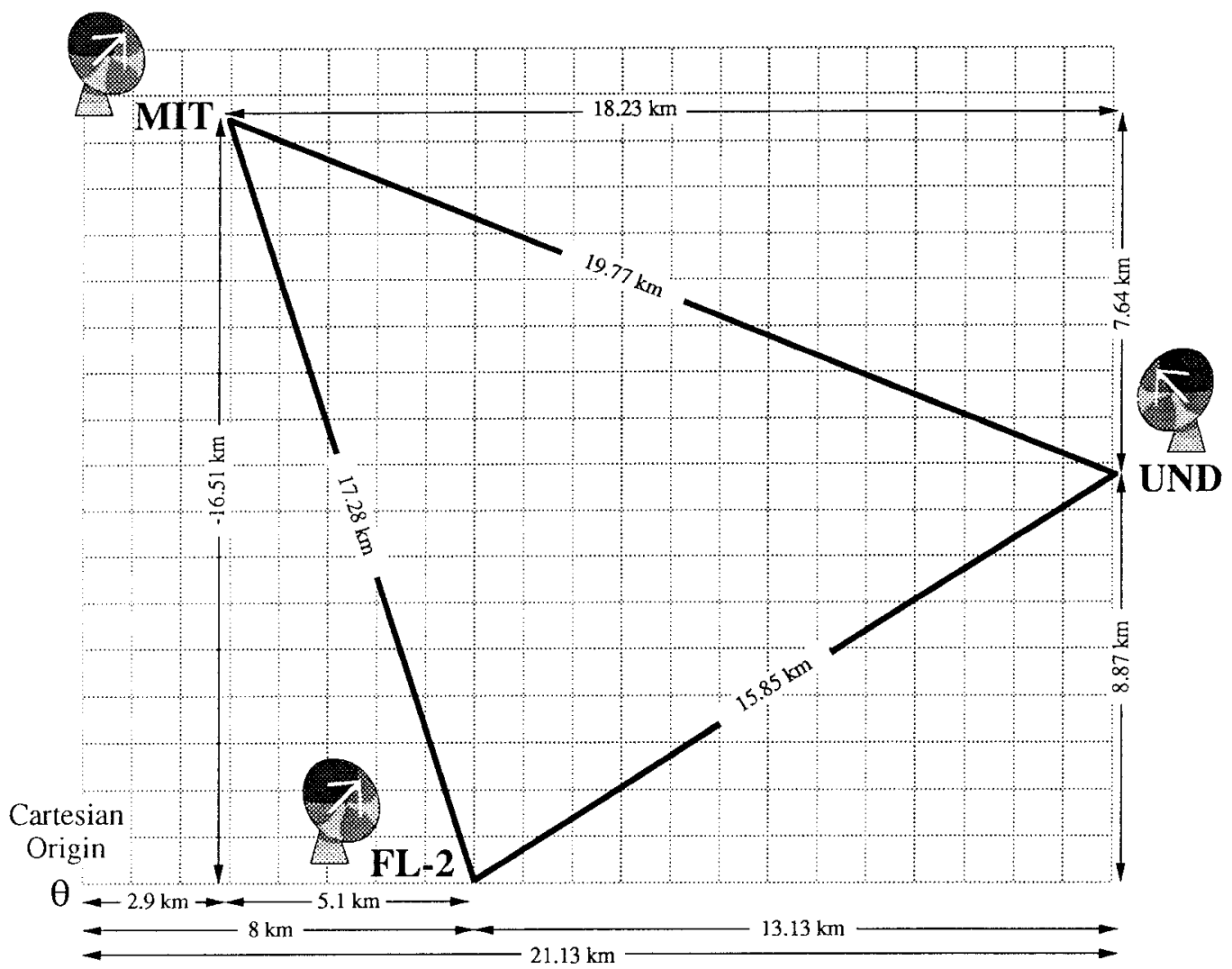

\section{Common origin for all 3 radars}

FIG. 1. Triple-Doppler network configuration. The data were interpolated onto a Cartesian grid with a common grid origin, $\theta$, for all three radars (FL-2-Florida terminal Doppler weather radar, UND—University of North Dakota C-band Doppler radar, MIT—Massachusetts Institute of Technology C-band Doppler radar).

though this is a rather short time interval, we show in the next section that it was adequate for our needs.

\section{b. Meteorological aspects}

On 9 August 1991, a severe multicell storm complex traversed the center of the triple-Doppler network (described above). Moving from the northwest, the storms generated an intense surface outflow with mesonet winds approaching $45 \mathrm{~m} \mathrm{~s}^{-1}$ around 1943 UTC. The high winds, associated with a microburst, were the most severe event recorded by the project that season.

Figure 2 shows the vertical velocity w obtained from the hybrid wind synthesis (the synthesis winds are also referred to as "observed") at $z=5.1 \mathrm{~km}$ at 1923 UTC and 1947 UTC as the storm complex propagates through the domain over the 24-min period-a distance of approximately $11.5 \mathrm{~km}$. This corresponds to a propagation speed of nearly $8 \mathrm{~m} \mathrm{~s}^{-1}$. The multicell characteristics of the convection are quite evident owing to the presence of short-lived, multiple updraft centers. Maximum vertical velocities from the hybrid synthesis, about half that predicted from parcel theory, approach $15 \mathrm{~m} \mathrm{~s}^{-1}$. [See Lazarus (1996) for a more complete description of the hybrid synthesis data.] Because the convection enters the domain from the northwest, we choose our analysis domain to be shifted (to the west), from that shown in Fig. 1, such that in all the horizontal cross sections, the FL-2 radar lies at the center of the southernmost edge of the domain. This places the MIT radar, which is 16.5 $\mathrm{km}$ to the north and $5.1 \mathrm{~km}$ to the east of the FL-2 radar, northwest (and outside of the) analysis domain. The UND radar, which is located $13 \mathrm{~km}$ to the east and 8.9 $\mathrm{km}$ north of FL-2, lies just to the east of the analysis domain.

In addition to its high spatial and temporal resolution, this dataset offers a variety of convective-scale weather features including: deep convection, surface cold pool, 
heavy precipitation, microbursts, and a distinct cloudtop divergence signature. These features provide a robust test of the capabilities, limitations, and challenges that face SDVR. Before applying the ZG scheme to the Orlando dataset (sections 4 and 5), we briefly discuss the moving reference frame characteristics.

\section{Technique description}

\section{a. Mean flow retrieval}

In order to define a moving reference frame one must first retrieve the mean flow. As discussed previously however, there exists no unique way in which to obtain this mean flow. The $\mathrm{ZG}$ functional in which the time tendency of an observed tracer, namely the reflectivity, is minimized, is given as

$J \equiv \int_{\Omega}\left\{\mu\left[\frac{\partial Z}{\partial T}+U \frac{\partial Z}{\partial x}+V \frac{\partial Z}{\partial y}+\left(W+W_{t}\right) \frac{\partial Z}{\partial z}\right]^{2}\right\} d \hat{x} d t$,

where $U, V$, and $W$ are the mean flow velocities, the integration is over both space $d \hat{\mathbf{x}}(d x, d y, d z)$ and time $d t$ and $W_{t}$ is the fall speed of the precipitation (Kessler 1969). Here, $Z$ is the reflectivity factor in units of $d B Z$, and the weight $\mu$ is given as the inverse variance of the residual in the reflectivity conservation equation (ZG96; Liou 1999). The mean flow retrieval described by Eq. (1) assumes that reflectivity is conserved, which is accurate only in the absence of cloud/precipitation growth and decay, and turbulence. We employ the terminal fall speed relationship given by Shapiro et al. (1995) and do not attempt to test the sensitivity of $\mathrm{ZG}$ to this assumption. It is possible however that this relationship is a source of error-especially in the vertical velocity retrieval. A 3D analytic study of the impact of the terminal fall speed relationship would quantify such sensitivities but is beyond the scope of this paper.

The integration of Eq. (1) can be taken over the entire volume or over a limited portion of the domain. In the case of the former, the retrieved velocities $(U, V)$ are then an estimate of the storm motion for the entire multicell system. The distinction between this approach and one that allows $\Omega$ (and thus $U, V$ ) to vary as a function of space $(x, y, z)$, is quite important. As we will show later, the choice of an appropriate reference frame remains very much flow dependent. For the case of an idealized flow where two reflectivity maxima undergo pure translation in opposite directions (and at the same speed), a volume integration of Eq. (1) will yield a $U$ and $V$ of zero. We attempt to address the issue, in part, of what constitutes a representative domain by showing the results for two different domains used to estimate the mean translation velocities. Equation (1) assumes that the translation velocities are significantly larger than either the turbulent velocities or fluctuations in the mean velocity (e.g., Shapiro et
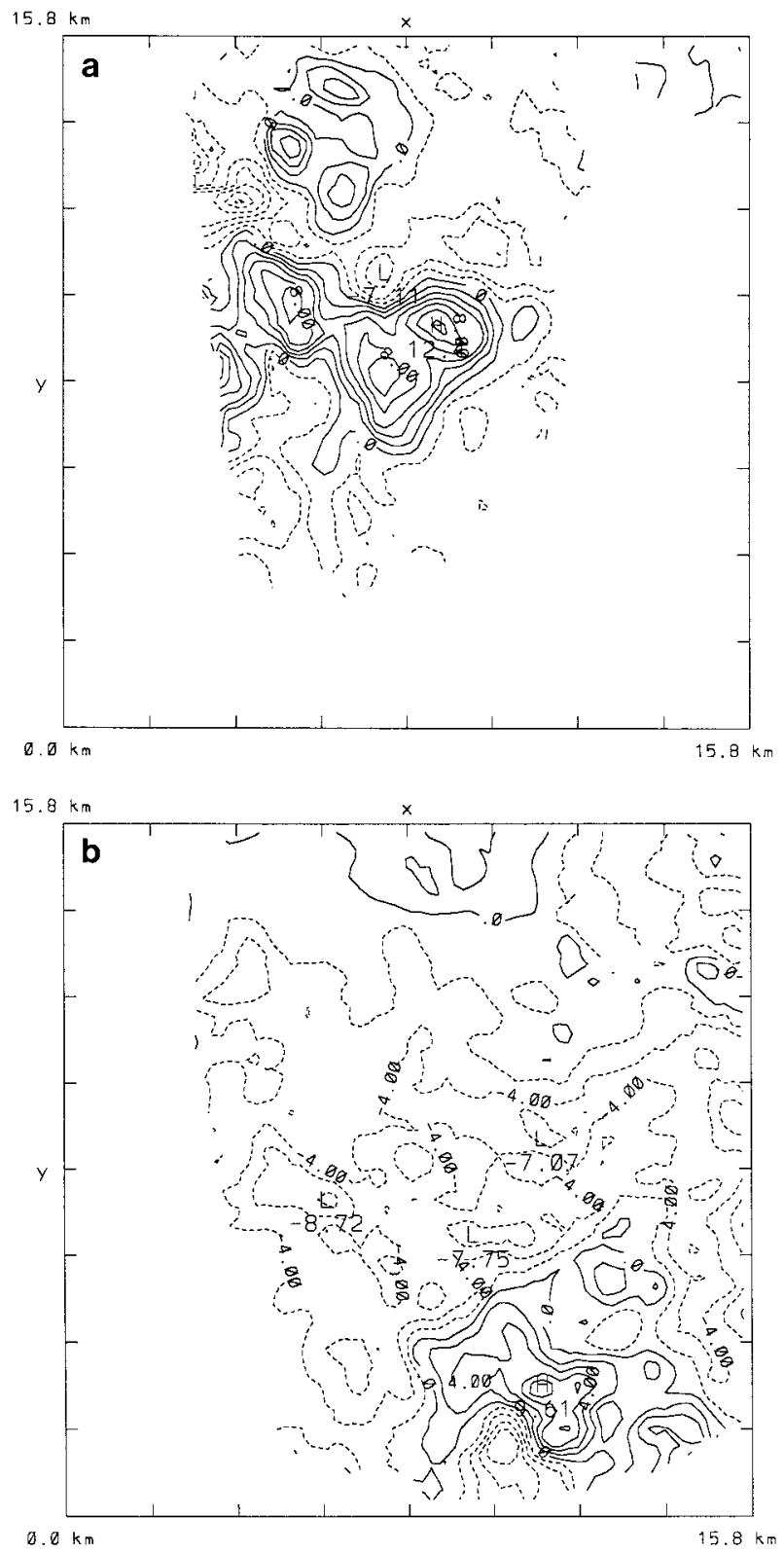

FIG. 2. Horizontal cross section of hybrid synthesis $w$ at $z=5.1$ $\mathrm{km}$ and (a) 1923:26 UTC, (b) 1947:49 UTC. The domain shown is $15.8 \mathrm{~km} \times 15.8 \mathrm{~km}$. The convection diminishes in intensity by 1947:49 UTC. The contour interval is $2.0 \mathrm{~m} \mathrm{~s}^{-1}$, dashed contours indicate negative values. FL-2 radar is located bottom center of figure.

al. 1995). It is precisely the failure of this hypothesis in the presence of vertical shear of the horizontal wind, that is, the observed reflectivity structures do not remain vertically coherent, that serves as the impetus for applying Eq. (1) as a function of height. These and other related issues will be discussed in section 4. [For a more complete discussion of the sensitivities of the ZG technique to variations in domain size see Lazarus et al. (1999).] Because of convergence problems, we 
take $\mu$ to be a constant, and thus it does not affect the minimum of the cost function in Eq. (1). Experiments where $\Omega$ is varied do indicate convergence if a sufficiently large number of grid points (on the order of 20) are used to estimate $\mu$. However, results from these experiments are not significantly different from those where $\mu$ is taken to be constant. To obtain the mean flow velocity from Eq. (1), we proceed as in $\mathrm{ZG}$ by minimizing Eq. (1) (assuming $W=0$ ), which results in a $3 \times 3$ matrix associated with the linear EulerLagrange equations.

\section{b. Perturbation flow retrieval}

Because the retrieval is best performed in a moving reference frame that minimizes errors in the time derivative of the reflectivity field (ZG96), once the mean flow is obtained, one can proceed to transform both the reflectivity and radial wind equations from a fixed frame to a moving frame \{see Liou [1999, Eq. (9)] for the transformation equations $\}$. Similar to the mean flow retrieval, the minimization of the moving frame (transformed) functional,

$$
J \equiv \int_{\hat{\mathbf{x}}, t}\left\{\mu^{\prime}\left(\frac{\partial Z}{\partial t^{\prime}}+u^{\prime} \frac{\partial Z}{\partial x^{\prime}}+v^{\prime} \frac{\partial Z}{\partial y^{\prime}}+\left(w^{\prime}+W_{t}\right) \frac{\partial Z}{\partial z^{\prime}}\right)^{2}+\left(v_{r}^{\prime}-u^{\prime} \frac{x^{\prime}-a^{\prime}}{r^{\prime}}-v^{\prime} \frac{y^{\prime}-b^{\prime}}{r^{\prime}}-w^{\prime} \frac{z^{\prime}-c^{\prime}}{r^{\prime}}\right)^{2}\right\} d \hat{\mathbf{x}}^{\prime} d t^{\prime}
$$

yields a system of three linear equations in three unknowns (i.e., the moving frame velocities, $u^{\prime}, v^{\prime}, w^{\prime}$ ). Here, $a^{\prime}=a-U \Delta t, b^{\prime}=b-V \Delta t$, and $c^{\prime}=c$ are the moving frame radar coordinates with respect to the radar location $(x, y, z)=(a, b, c), \Delta t$ is the difference between the observation time and some reference time (i.e., the time the retrieval is valid), $d x^{\prime}$ is area of integration in the moving frame, and $\mu^{\prime}$ and $r^{\prime}$ are the moving frame weight and radar-to-gridpoint distance, respectively (see GZ96 for details). The total wind field $(u, v, w)$ is then obtained by combining the mean $(U, V)$ and perturbation winds $\left(u^{\prime}, v^{\prime}, w^{\prime}\right)$. Estimates of the mean vertical velocity, which are nonzero for the retrievals performed herein, are not used to transform the reflectivity and radial wind equations to a moving reference frame. This will likely contribute to retrieval error as proper minimization of the reflectivity time derivative depends on all three mean wind components. In practice, one might expect $W$ to be small as the largescale vertical motion is typically two orders of magnitude smaller than that of the deep convection. In this paper, we concentrate our efforts on the moving frame as defined by the mean horizontal components only. It would, however, be instructive to examine the impact of this assumption (i.e., $W=0$ ) on the retrieval.

Because the radial wind conservation equation is employed as a weak constraint, the retrieved radial winds produced by this will not "exactly" match the observed radial wind. However, because the velocity stationarity assumption is likely violated [e.g., Lazarus et al. (1999, their Fig. 11)], it makes sense to choose a weak constraint on $v_{r}$. In the absence of reflectivity error, Lazarus et al. (1999) show that the reflectivity conservation constraint is essential for the retrieval of the local flow velocities, that is, radial wind information alone is not sufficient to determine the local flow. This is somewhat intuitive as if the radial wind were sufficient to deter- mine the local flow, then a simple projection of $v_{r}$ onto the Cartesian components would suffice. Because of the idealized nature of these results, it is difficult to ascertain what the impact would be for imperfect data. Ideally, if the weight in Eq. (1) is an accurate indicator of the amount of radial wind/reflectivity error, the two will be weighted appropriately. Additionally, the reflectivity conservation assumption will, at times, be violated for deep convection-especially if it is rapidly evolving. Thus, assuming "perfect" reflectivity (i.e., a strong constraint) may also be a source of retrieval error [see Lazarus et al. (1999) for a discussion of the impact of reflectivity errors in the presence of perfect radial winds].

\section{Sensitivity tests}

The ZG methodology described in sections $3 \mathrm{a}$ and $3 \mathrm{~b}$ is applied to the deep convective storm discussed in section 2. Although single-Doppler radar data were available from all three radars, only data from FL-2 radar (see Fig. 1) were used in our retrieval experiments. This choice was purely arbitrary.

\section{a. Statistics}

The retrievals are performed on a nonstaggered grid, that is, all variables are collocated with the radial velocity and reflectivity. Grid spacings of $\Delta x, \Delta y=400$ $\mathrm{m}$, and $\Delta z=200 \mathrm{~m}$ are used in the sensitivity tests discussed in this section. The results are quantified by comparing the retrieved $3 \mathrm{D}$ velocity with the hybrid wind synthesis via the volume rms error statistic; that is,

$$
\mathrm{rms}=\sum \sqrt{\frac{\left(\chi^{\mathrm{obs}}-\chi^{\mathrm{ret}}\right)^{2}}{N}}
$$

the mean flow statistic: 


$$
\bar{\chi}=\frac{1}{N} \sum \chi
$$

and Spearman's rank correlation coefficient:

$$
\rho\left(\chi^{\mathrm{obs}}, \chi^{\mathrm{ret}}\right)=\frac{\sum\left(\chi^{\mathrm{obs}}-\bar{\chi}^{\mathrm{obs}}\right)\left(\chi^{\mathrm{ret}}-\bar{\chi}^{\mathrm{ret}}\right)}{\sqrt{\sum\left(\chi^{\mathrm{obs}}-\bar{\chi}^{\mathrm{obs}}\right)^{2} \sum\left(\chi^{\mathrm{ret}}-\bar{\chi}^{\mathrm{ret}}\right)^{2}}},
$$

where $\chi^{\text {obs }}$ and $\chi^{\text {ret }}$ are the observed and retrieved velocities. Here $N$ is the total number of points in the area where the retrieved and hybrid synthesis winds coincide.

Although the ZG scheme retrieves the Cartesian wind components $(u, v, w)$ directly, these components consist of both the observed (i.e., $\boldsymbol{v}_{r}^{\text {obs }}$ ) and unobserved (i.e., tangential $v_{\phi}^{\text {ret }}$ and polar $\left.v_{\theta}^{\text {ret }}\right)$ winds. Because we want to examine the component of the retrieved wind that is completely unobserved, we seek to map the retrieved Cartesian winds $\left(u^{\text {ret }}, v^{\text {ret }}\right)$ back to their unobserved spherical counterpart $\left(v_{\phi}^{\text {ret }}\right)$. This is a relatively straightforward procedure and can be accomplished by simply projecting the Cartesian velocity vector $(\mathbf{V})$ onto the tangential $\left(\hat{\mathbf{e}}_{\phi}\right)$ unit vector, which yields an expression for the azimuthal velocity component in terms of its Cartesian counterparts; that is,

$$
v_{\phi}=\frac{1}{\sqrt{x^{2}+y^{2}}}(u y-v x) .
$$

We use Eq. (6) to sample both the hybrid synthesis Cartesian components ( $\left.u^{\text {obs }}, v^{\text {obs }}\right)$ to obtain $v_{\phi}^{\text {obs }}$, and the retrieved Cartesian components $\left(u^{\text {ret }}, v^{\text {ret }}\right)$ to obtain $v_{\phi}^{\text {ret }}$. The $v_{\phi}^{\text {obs }}$ and $v_{\phi}^{\text {ret }}$ are then used in Eqs. (10)-(12) to calculate the various statistics. Note that when applying Eq. (6), we must take into account the radar location.

\section{b. Analysis error}

Because we consider the hybrid synthesis data to be the "truth" (i.e., we use it to validate the retrievals), it is important to recognize the inexact nature of the analysis technique used to produce the three-dimensional wind field from the single-Doppler data. Additionally, errors in the radial velocity measurements also feed into the analysis. As a result, the verification provided by the hybrid synthesis dataset deserves some scrutiny. In particular, we consider the extent to which the velocity field from the hybrid synthesis, when projected onto the radial from a given radar, agrees with the actual radial wind observed by the same radar. The radial wind component can be written in terms of Cartesian components as

$$
v_{r}=u \frac{x}{r}+v \frac{y}{r}+w \frac{z}{r}
$$

We apply Eq. (7) to compare the observed radial wind component sampled from the synthesized Doppler Cartesian components against the radial winds observed directly by FL-2. Figure 3 shows the rms differences

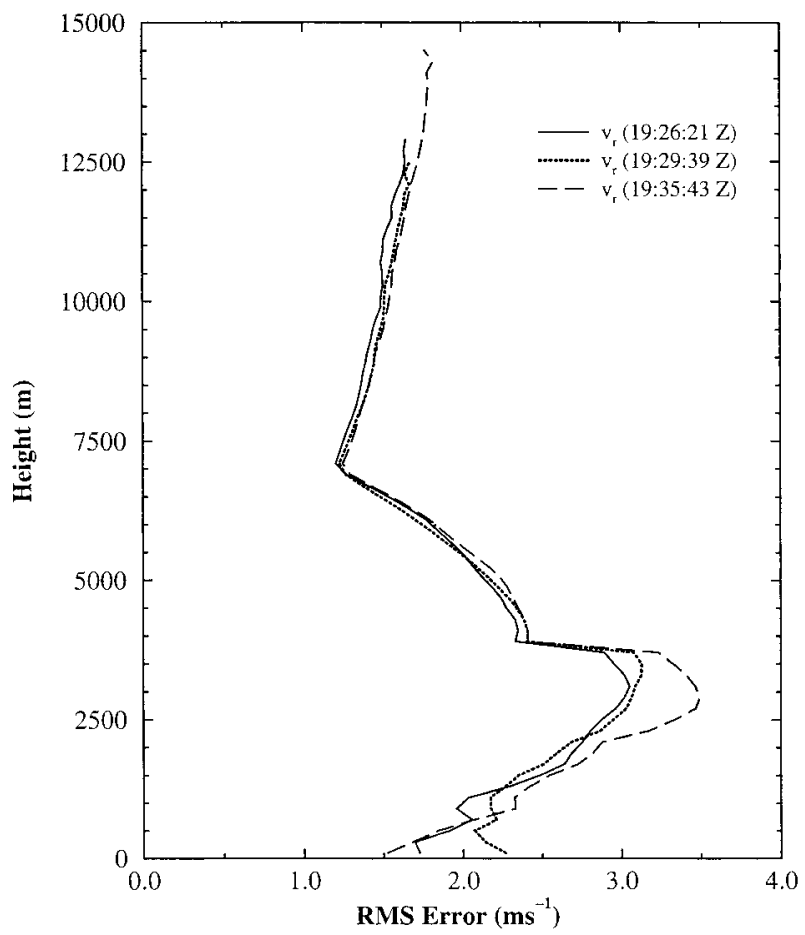

FIG. 3. The $z$ profile of the rms error between the FL-2 observed radial wind and the radial wind sampled from the hybrid wind analysis at 1926:21, 1929:39, and 1935:43 UTC.

between these two fields as a function of height for volume scans taken at 1926:21, 1929:39, and 1935:43. Even though the radial velocities from each of the three radars are used to perform the hybrid synthesis, there are inherent and systematic discrepancies. In fact, the rms error approaches $3.5 \mathrm{~m} \mathrm{~s}^{-1}$ near $3 \mathrm{~km}$. Despite these discrepancies, high elevation radial winds measured by the FL-2 radar support the hybrid synthesis estimates of the vertical velocity. Additionally, the hybrid synthesis estimates of the vertical velocity are comparable to that reported by Yuter and Houze (1995) for a similar convective event on the Florida peninsula just a few days after the event used herein. Note also that the close proximity of the radars (the baselines are less than 20 $\mathrm{km}$ ) and the features of interest propagating through the center of the radar network will greatly reduce synthesized vertical velocity errors [see DeLaura et al. (1989) for more details on the hybrid analysis]. In light of the aforementioned problems, the hybrid synthesis still represents the best guess of the $3 \mathrm{D}$ wind field.

\section{c. Reference frame experiments}

In the following three experiments, three volume scans taken at 1923:36, 1926:21, and 1929:39 are used to perform the retrieval. The reference time is taken to be the 1926:21 dataset, that is, this is the time at which the retrieval is valid. Note that at least two volume scans are needed to estimate the reflectivity time derivative 
TABLE 1. Statistical comparison between the SDVR and hybrid wind synthesis data for experiments with varying reference frames.

\begin{tabular}{lcccccr}
\hline \hline Exp. & $v_{\phi}$ rms & $v_{\phi}(\rho)$ & $\bar{v}_{\phi}$ & $v_{r}$ rms & $v_{r}(\rho)$ & $\bar{v}_{r}$ \\
\hline OBS & & & 0.793 & & & -2.120 \\
NMV & 3.022 & 0.452 & 1.003 & 4.040 & 0.619 & 0.732 \\
MV & 3.071 & 0.496 & 1.452 & 3.754 & 0.698 & 0.383 \\
LVL & 3.159 & 0.535 & 0.879 & 3.667 & 0.702 & 0.330 \\
\hline
\end{tabular}

but the algorithm does not require that the volume scans be evenly spaced in time. We performed experiments where the number of volume scans were varied from two to four. The results of these experiments (not shown here) are consistent with those of Xu et al. (1994a,b) and $\mathrm{Xu}$ et al. (1995) and ZG96-showing an improved cross-beam wind retrieval with increasing number of volume scans. With the exception of experiment LVL in Table 1, the experiments performed in the moving reference frame are performed using a mean flow determined by a volume integration of Eq. (1). Note that we use the observed radial wind rather than that sampled from the hybrid synthesis to calculate the $v_{r}$ rms statistics. Consequently, the interpretation of the error statistics for the radial component are somewhat more straightforward than those for the tangential component. However, if one assumes that the synthesis errors in the cross-beam component obtained from the hybrid technique are comparable to the radial wind errors shown in Fig. 3 (the best-case scenario), then errors in the retrieved tangential component can be interpreted in a similar manner as its radial wind counterpart. Unfortunately, in the latter case, it is impossible to separate the error contribution from the hybrid synthesis from that of the retrieval.

In theory, the perturbation flow can be retrieved using a single point. Yet because observation errors can lead to matrix ill-conditioning for the linear system of equations, it is necessary to involve neighboring points in the calculation of the perturbation flow (ZG96). The addition of these surrounding points, which define the area of integration $\left[d \hat{x}^{\prime}\right.$, Eq. (2)], has a stabilizing effect on the solution but does so at the expense of potentially removing detail from the retrieved wind field. Carbone et al. (1985) have demonstrated that, at close range, one needs at least 6-8 radar samples to resolve a complete wave. For the following experiments we choose a $d \hat{x}^{\prime}$ of $4 \Delta$ (where $\Delta=400 \mathrm{~m}$ represents only the $x$ and $y$ directions). We performed experiments whereby $d \hat{x}^{\prime}$ was varied but for the sake of brevity these results are not presented here. Our choice of $d \hat{x}^{\prime}$ yields a sufficient number of surrounding points such that the solution of perturbation flow equation is stable, preserves as much detail in the retrieved winds as possible, and resolves the features of interest.

The results of three experiments are presented in Table 1 . Also shown are the hybrid synthesis mean radial and tangential wind components (OBS). Experiment NMV is performed in a nonmoving reference frame while experiment MV is performed in a moving reference frame where the mean flow is determined by a volume integration of Eq. (1). LVL denotes a moving frame retrieval where the mean flow is instead determined as a function of height (i.e., the mean flow is retrieved level-by-level). A plot of the rms error as a function of height reveals that LVL notably out performs NMV both near the surface and aloft (Fig. 5). Near the surface, the tangential component rms error for LVL is approximately $2.0 \mathrm{~m} \mathrm{~s}^{-1}$ less than $\mathrm{NMV}$, while at storm top the improvement is approximately $5 \mathrm{~m} \mathrm{~s}^{-1}$. The uniform moving frame (MV) lies between LVL and NMV in the lowest $1 \mathrm{~km}$ and is actually worse than the nonmoving frame near the domain top. Between the surface and storm top, the three reference frames perform comparably with the exception of the spike in LVL around $10 \mathrm{~km}$. We believe that this spike is, in part, a result of our resolution (i.e., data sampling) as it is present in the $200-\mathrm{m}$ retrieval but is less significant (figure not shown). The performance of LVL is tied to the particular mean flow at a given level, that is, if there is weak mean flow at that level then there will be no benefit (or even possibly a detriment) using the height-dependent reference frame. For the numerous experiments we performed (but do not show herein), the statistics of the uniform mean flow reference frame are close to that of the nonmoving reference frame (for this case). We believe this occurs because domain (i.e., volume) estimates of $U, V$ are quite small (in many of our retrievals, the volume mean flow estimates for $U$ were typically less than $1.5 \mathrm{~m} \mathrm{~s}^{-1}$ and $V$ was near $0.0 \mathrm{~m} \mathrm{~s}^{-1}$ ). Overall, results for the uniform frame are mixed. For example, rms errors for the uniform frame were better than the nonmoving frame when the actual mean flow at the level was close to that estimated using a volume mean estimate for $U$ and $V$. This finding is somewhat intuitive. For example, imagine a unidirectional shear profile with a critical level (i.e., zero mean wind) that yields a zero mean flow in the uniform reference frame. In this case, we might expect a poor performance for the uniform frame everywhere except in the midlevels, while the LVL frame should perform better at all levels except for the critical level.

These results illustrate the potential importance concerning the definition of a moving reference frame. For this particular case a volume estimate of the mean flow is not "optimal" and, in the presence of vertical shear in the horizontal wind (Fig. 4), it is better to define a moving reference frame that is height dependent. Improving the retrieval at these levels may be critical for hazard warnings as well as for supplying fields to initialize numerical forecasts-especially because of the surface outflow (Lazarus 1996), which plays an important role in the convective initiation and sustenance problem for multicell convection. Also note that the mean of the retrieved tangential component agrees quite well with observations, and that $\rho$ is largest for experiment LVL. The larger volume rms in LVL is a result 


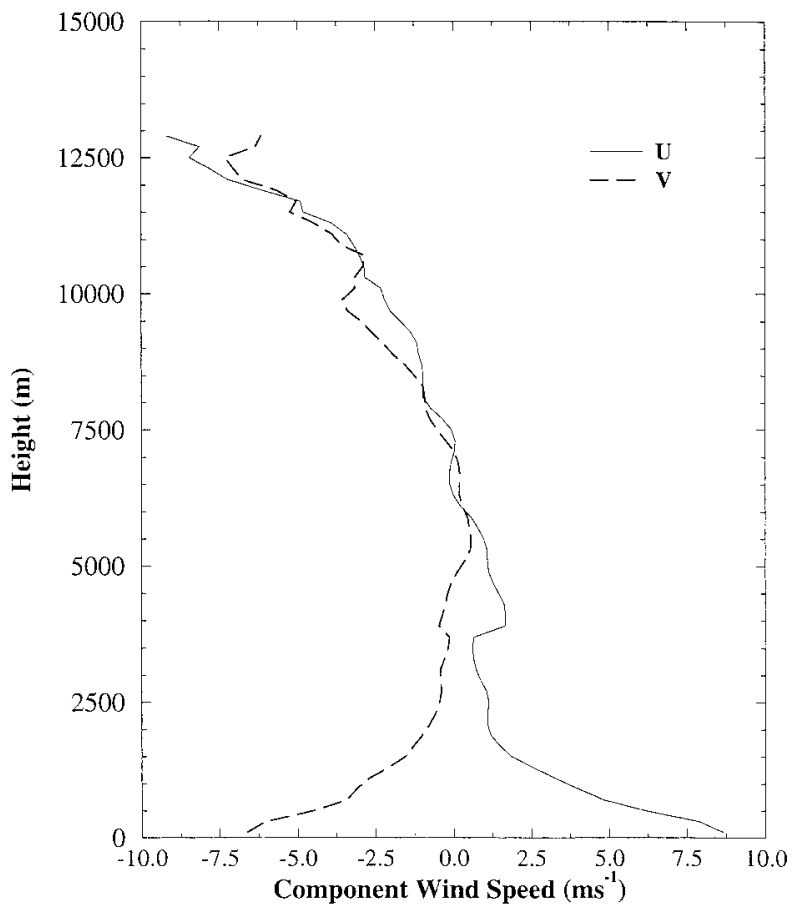

FIG. 4. The $z$ profile of the observed mean wind $u$ component (solid curve) and $v$ component (dashed curve) at 1926:21 UTC from the hybrid wind synthesis data.

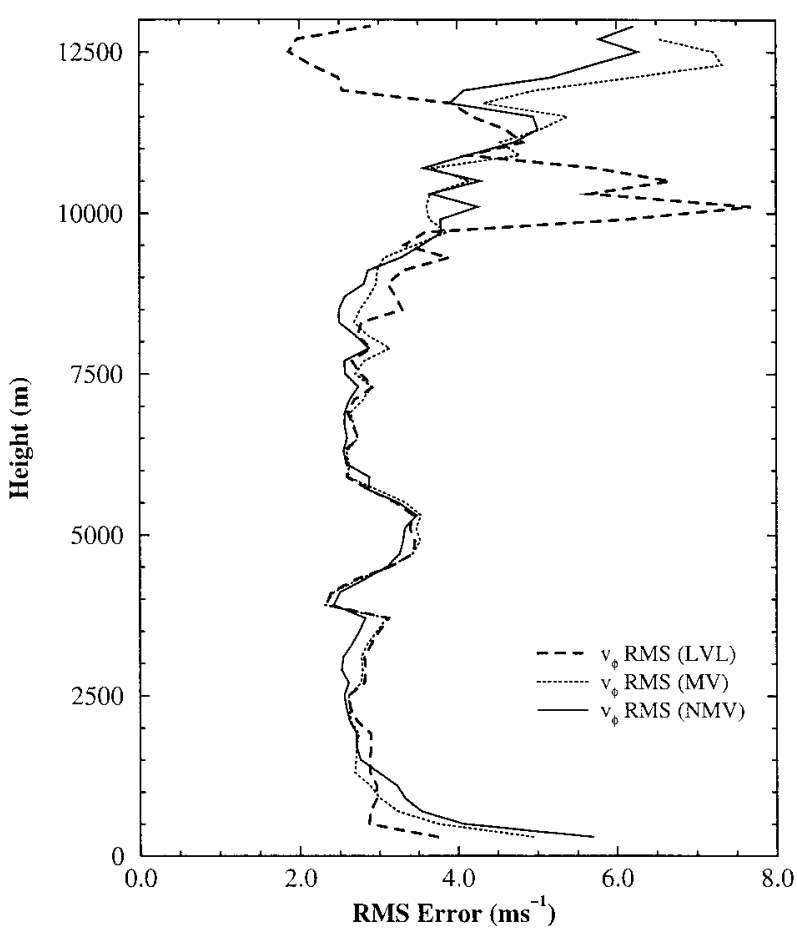

FIG. 5. The $z$ profile of the rms error in the tangential wind component for the nonmoving (solid curve), uniform moving (dotted curve), and level-by-level (dashed curve) reference frames at 1926: 21 UTC. The profiles are taken from the sensitivity tests NMV, MV and LVL in Table 1.

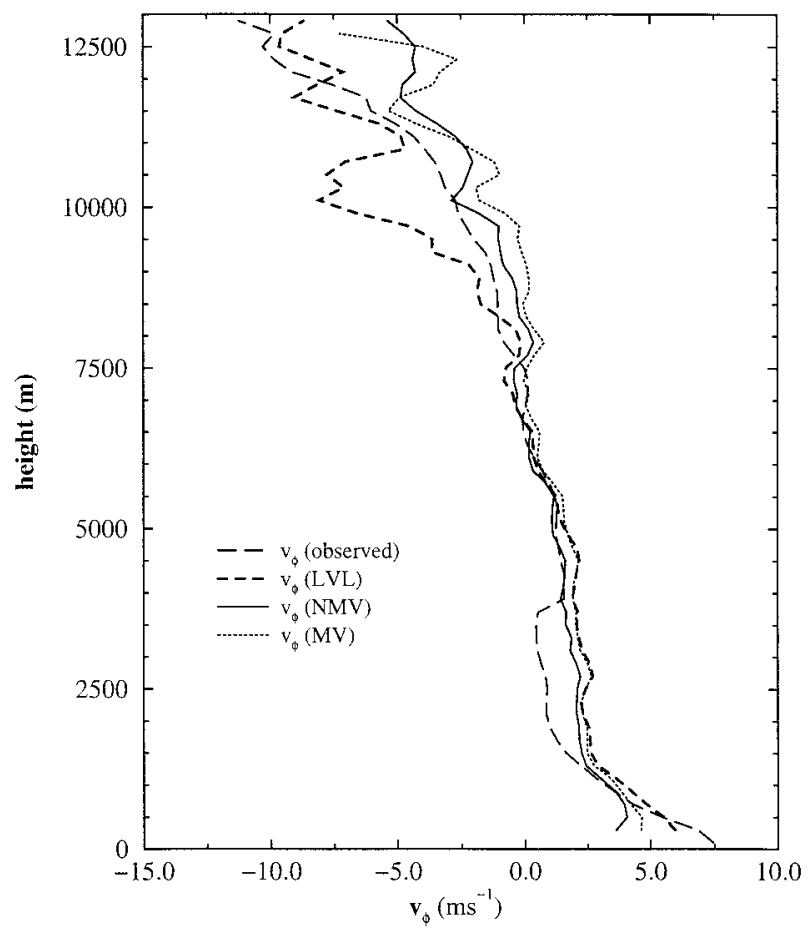

FIG. 6. The $z$ profile of the mean tangential wind component at 1926:21 UTC for the observations (long dashed curve), nonmoving (solid curve), uniform moving (dotted), and level-by-level (short dashed curve) reference frames. Note the improvement above and below for the moving reference frame. The profiles are taken from the sensitivity tests NMV, MV, and LVL in Table 1.

of the "spike" near $10 \mathrm{~km}$ (see Fig. 5). Figure 6, which depicts the mean profiles for the observed and retrieved tangential velocity, reveals that the LVL overestimates (by approximately $5 \mathrm{~m} \mathrm{~s}^{-1}$ ) the magnitude of the crossbeam component at this level. A similar "spike" is present in NMV as well but is closer to that observed.

\section{Tangential velocity retrieval}

In this section, we focus on the results of one particular retrieval that is valid at reference time 1935:43 UTC. For the sake of brevity, we refer only to this retrieval (R35) while the statistics associated with two comparable retrievals, valid at reference times 1926:21 and 1929:39 (R26 and R29), are displayed for comparison purposes in Table 2 and discussed in section $5 \mathrm{~b}$.

We choose a $d \hat{x}^{\prime}$ of $6 \Delta$ as we perform these retrievals using the radar data at its highest resolution (i.e., an isotropic grid with $\Delta=200 \mathrm{~m}$ ). As discussed previously, this choice reflects a compromise between solution stability, the filtering of small-scale noise (unresolvable features, random data errors, etc.), and a successful retrieval of the resolvable-scale winds. As in the sensitivity tests in section 4, the single-Doppler data were obtained from the FL-2 radar. However, we use four volume scans for R35 instead of three as in the sensitivity experiments. Figure 7 shows the reference 
TABLE 2. Statistical comparison between the SDVR and hybrid wind synthesis data for all three retrievals.

\begin{tabular}{|c|c|c|c|c|c|c|c|c|c|c|}
\hline Retr & $v_{\phi} \mathrm{rms}$ & $v_{\phi}(\rho)$ & $\bar{v}_{\phi} \operatorname{Retr}$ & $\bar{v}_{\phi} \mathrm{Obs}$ & $v_{r} \mathrm{rms}$ & $v_{r}(\rho)$ & $\bar{v}_{r} \operatorname{Retr}$ & $\bar{v}_{r} \mathrm{Obs}$ & $* v_{r} \mathrm{rms}$ & $* \bar{v}_{r} \operatorname{Retr}$ \\
\hline R26 & 2.93 & 0.573 & 0.829 & 0.839 & 3.82 & 0.686 & 0.47 & -2.11 & 2.78 & -1.19 \\
\hline R29 & 3.10 & 0.566 & 0.662 & 0.817 & 3.87 & 0.723 & 0.48 & -1.96 & 3.22 & -1.21 \\
\hline $\mathrm{R} 35$ & 2.90 & 0.773 & 0.214 & 0.289 & 4.26 & 0.700 & 0.53 & -2.12 & 3.67 & -0.98 \\
\hline
\end{tabular}

times given in bold for all three retrievals. Although as few as two volume scans would suffice for each retrieval, we use four volume scans here. The missing data at 1932:25 do not pose a logistical problem in that the ZG retrieval can accommodate missing or irregularly spaced data (in time or space). The use of an increased number of volume scans yields slightly improved retrievals. It is important to point out however that this may not always be the case as an increased number of volume scans increases the likelihood that the reflectivity conservation constraint will be violated. We feel however that the time resolution of our dataset is sufficient to avoid, for the most part, any significant errors associated with the velocity stationarity assumption. Problems associated with sparse data can be overcome, more appropriately, by introducing a smoothing function directly into the ZG functional (e.g., Liou 1999).

In section 5a, we present and compare the results of R35 in both the nonmoving and moving reference frames (where the mean flow is determined as a function of height, e.g., LVL). In section $5 b$ we compare the volume-averaged statistics from R35 with those obtained from R26 and R29.

\section{a. $R 35$}

R35 illustrates the importance of the moving reference frame as defined by integrating Eq. (1) as a function of height. Figure 8 depicts the horizontal cross section of the tangential wind component at $z=0.3 \mathrm{~km}$ for the hybrid Doppler observations and nonmoving and moving reference frames, respectively. (This is the lowest level for which the retrieval is performed.) The contour interval is $2.0 \mathrm{~m} \mathrm{~s}^{-1}$ in all cross sections unless otherwise stated. Although data exists as low as $100 \mathrm{~m}$, centered spatial differences preclude the use of the lowest level because the reflectivity is not known below this level. It is important to point out that the retrieval of the tangential component represents a severe test of the
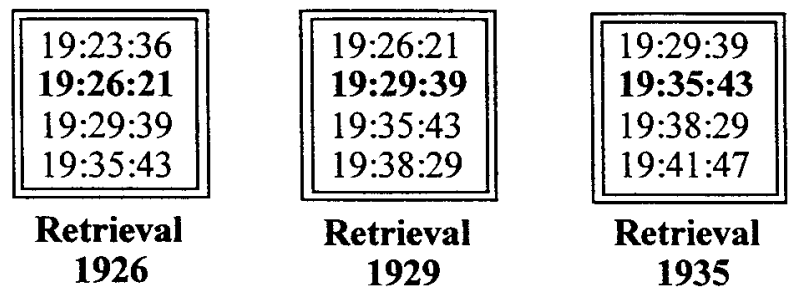

FIG. 7. Schematic diagram indicating the single-Doppler volume scans that are used for the retrievals. The time in bold indicates the retrieval reference time.
SDVR as this component is completely unobserved. The retrieved moving frame $v_{\phi}$ maximum $\left(21.7 \mathrm{~m} \mathrm{~s}^{-1}\right)$ is relatively close to that observed $\left(25.8 \mathrm{~m} \mathrm{~s}^{-1}\right)$, while the nonmoving frame significantly underestimates the maximum $v_{\phi}\left(7.6 \mathrm{~m} \mathrm{~s}^{-1}\right)$. The retrieved maximum is associated with a feature in the eastern portion of the domain. To the east of this feature, the moving frame recovers another region where the retrieved $v_{\phi}$ is quite close to the maximum. The location of this second smaller feature agrees quite nicely with the observed maximum. Neither the stationary nor moving frame retrievals recover the region to the west where $v_{\phi}<0$.

Because the convection extends over a depth of 14 $\mathrm{km}$, it is important to evaluate the retrieval performance at higher elevations as well. Figure 9 is the same as Fig. 8 , except at $z=8.3 \mathrm{~km}$. This level was selected somewhat arbitrarily but is representative of the retrieval performance in the midlevels of the multicell convection. At this level, the moving and nonmoving frames perform equally well-each captures the overall divergence pattern of $v_{\phi}$. The mean flow velocities, $U$ and $V$ [as determined from (1)], are $0.6 \mathrm{~m} \mathrm{~s}^{-1}$ and $-1.0 \mathrm{~m} \mathrm{~s}^{-1}$, respectively, compared to $5.1 \mathrm{~m} \mathrm{~s}^{-1}$ and $-1.8 \mathrm{~m} \mathrm{~s}^{-1}$ at $z=0.3 \mathrm{~km}$. The reduced mean flow at $z=8.3 \mathrm{~km}$ suggests that the moving frame does not benefit the retrieval at this level compared to the near-surface retrieval where the mean winds are stronger (a direct result of the cold pool). In the case where the mean flow is relatively small, such as $z=8.3 \mathrm{~km}$, interpolation errors encountered as a result of shifting the coordinate system may actually overwhelm the error reduction due to minimization of the reflectivity time derivative.

The retrieved stationary frame minimum $(-3.6 \mathrm{~m}$ $\left.\mathrm{s}^{-1}\right)$ is close to the moving frame minimum $(-3.1 \mathrm{~m}$ $\left.\mathrm{s}^{-1}\right)$ and both are quite a bit less than observed $(-12.0$ $\left.\mathrm{m} \mathrm{s}^{-1}\right)$. The retrieved stationary frame maximum $(3.5$ $\mathrm{m} \mathrm{s}^{-1}$ ) and moving frame maximum $\left(4.6 \mathrm{~m} \mathrm{~s}^{-1}\right)$ are somewhat less than the observed $\left(6.0 \mathrm{~m} \mathrm{~s}^{-1}\right)$. The $v_{\phi}$ couplet in the northeast portion of the domain is retrieved quite well in both reference frames, and each recovers a weak couplet near the southern edge as well as a region (running southeast to northwest) of relative maximum $v_{\phi}$. Despite some agreement with the observations, the retrieval limitations are obvious, as the small-scale features in the observations are not recovered in either reference frame retrieval. Time and space integrations stabilize the least squares problem via averaging, yet a direct result of this filtering is the removal of both small-scale signal and noise. Liou (1999) has shown that the addition of mass conservation into the 

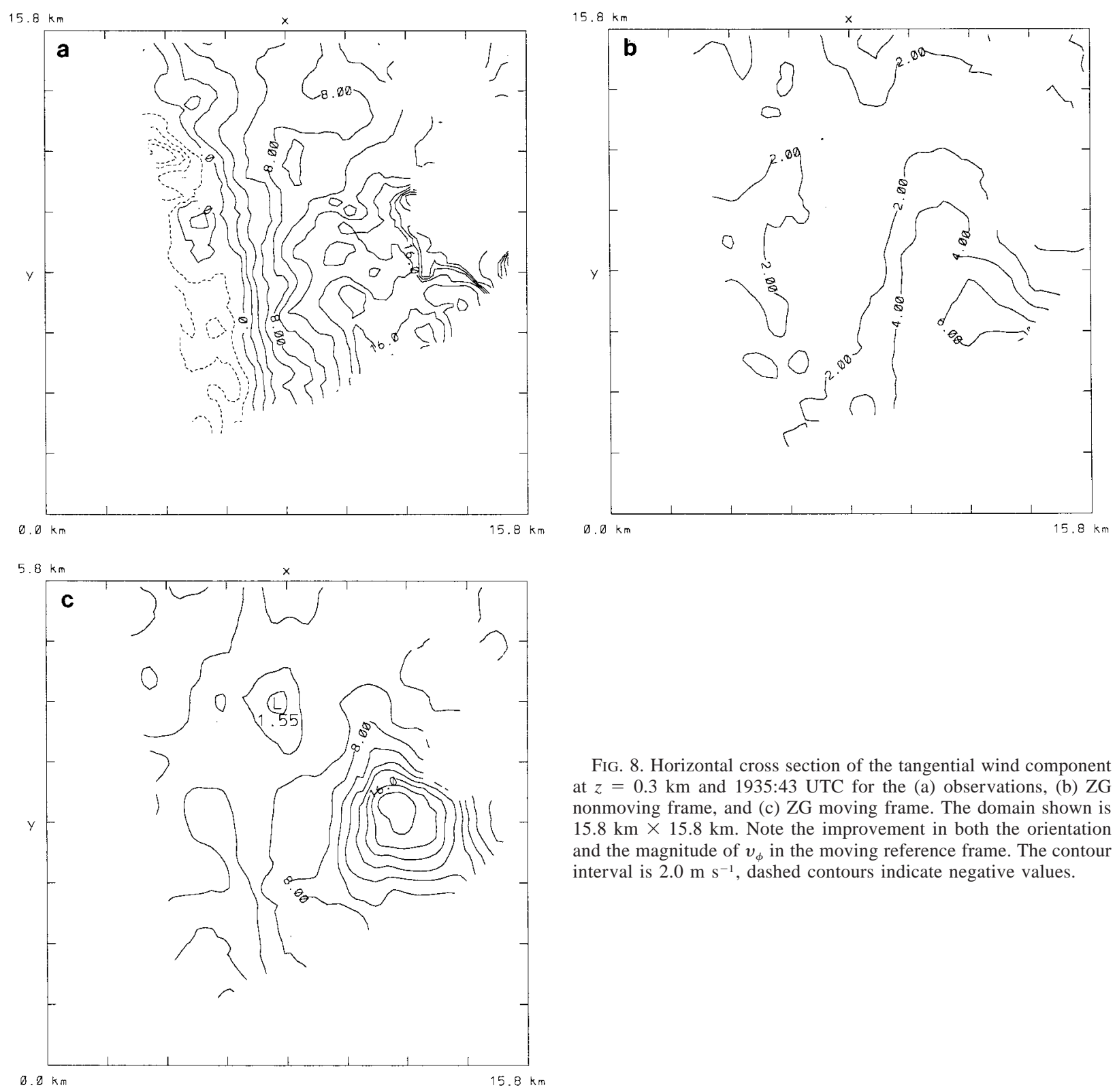

FIG. 8. Horizontal cross section of the tangential wind component at $z=0.3 \mathrm{~km}$ and 1935:43 UTC for the (a) observations, (b) ZG nonmoving frame, and (c) ZG moving frame. The domain shown is $15.8 \mathrm{~km} \times 15.8 \mathrm{~km}$. Note the improvement in both the orientation and the magnitude of $v_{\phi}$ in the moving reference frame. The contour interval is $2.0 \mathrm{~m} \mathrm{~s}^{-1}$, dashed contours indicate negative values.

cost function [Eq. (1)] can significantly improve the ZG retrieval at low elevation angles.

An east-west vertical cross section $4.8 \mathrm{~km}$ north of the FL-2 radar is shown in Fig. 10 for the observed, nonmoving, and moving reference frame calculations of $v_{\phi}$. The moving frame retrieval performs relatively well over the entire domain. While results in the midlevels are not significantly different for the two reference frames, there are somewhat noticeable differences both near the surface and domain top. The low-level gust front, identified by the relatively large vertical $v_{\phi}$ gradient in the boundary layer of the observations (Fig. 10a), is virtually nonexistent in the stationary frame (Fig. 10b), yet is visible in the moving frame (Fig. 10c) - extending to a depth similar to that observed (approximately $1.5 \mathrm{~km})$. The maximum tangential component for the observations $\left(17.6 \mathrm{~m} \mathrm{~s}^{-1}\right)$ and moving frame $\left(12.2 \mathrm{~m} \mathrm{~s}^{-1}\right)$ occurs in the cold pool, while that for the stationary frame $\left(6.8 \mathrm{~m} \mathrm{~s}^{-1}\right)$ occurs just above the surface outflow. The minimum tangential component occurs in the upper portions for the observations $(-16.9$ $\left.\mathrm{m} \mathrm{s}^{-1}\right)$, moving frame $\left(-15.0 \mathrm{~m} \mathrm{~s}^{-1}\right)$, and stationary frame $\left(-5.4 \mathrm{~m} \mathrm{~s}^{-1}\right)$. Note the significant difference between the moving frame and stationary frame retrievals as the stationary frame $v_{\phi}$ has the wrong sign (i.e., $>0$ ) near the domain top. At midlevels, the moving and stationary frames are in good agreement with the observations as a region where $v_{\phi}$ is less than zero divides 

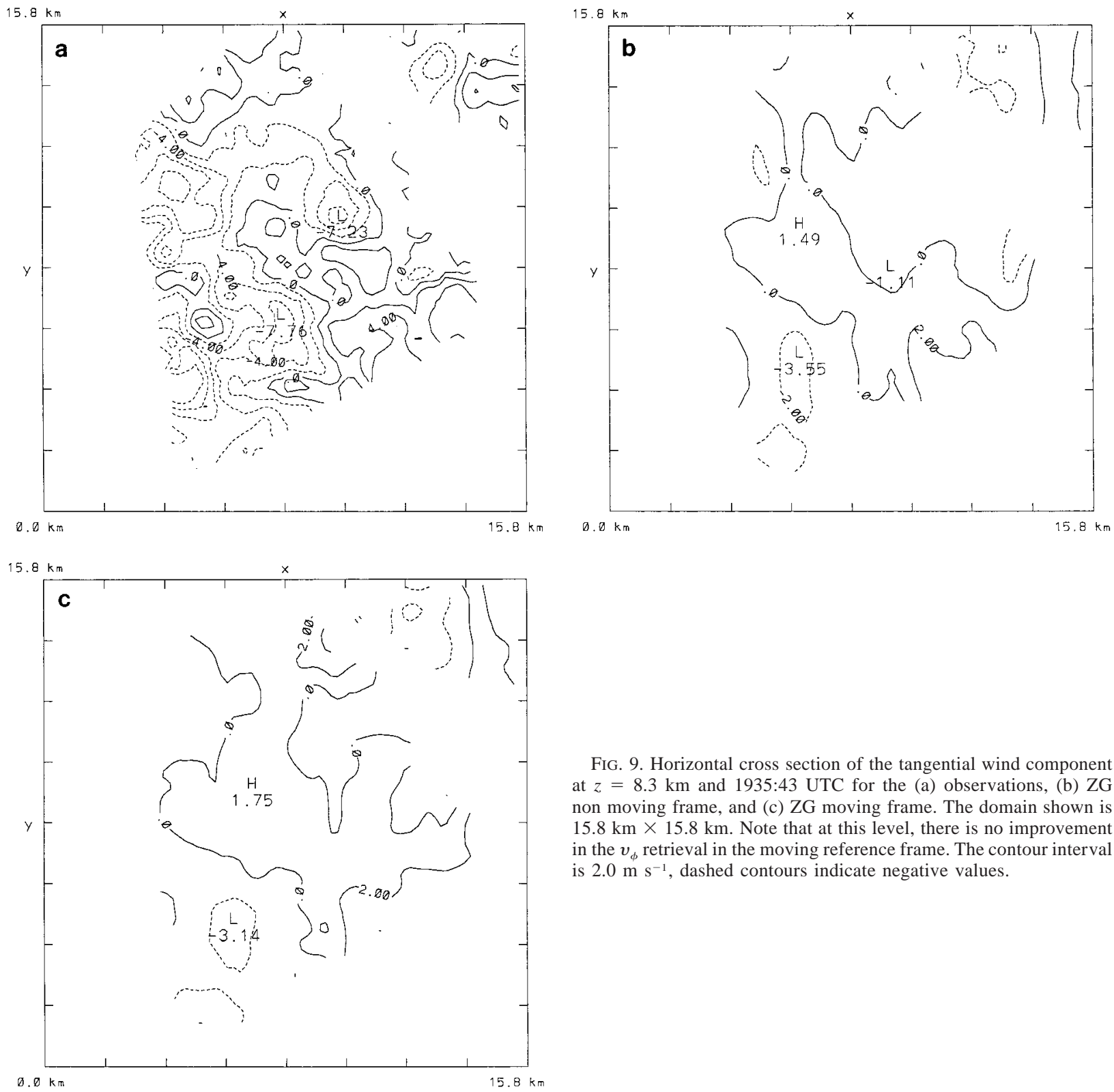

FIG. 9. Horizontal cross section of the tangential wind component at $z=8.3 \mathrm{~km}$ and 1935:43 UTC for the (a) observations, (b) ZG non moving frame, and (c) ZG moving frame. The domain shown is $15.8 \mathrm{~km} \times 15.8 \mathrm{~km}$. Note that at this level, there is no improvement in the $v_{\phi}$ retrieval in the moving reference frame. The contour interval is $2.0 \mathrm{~m} \mathrm{~s}^{-1}$, dashed contours indicate negative values.

regions to the east and west where $v_{\phi}$ is greater than zero. The moving frame does not retrieve the full magnitude of the tangential component in these regions, but does exhibit a relative minimum in both areas.

Figure 11 shows the rms errors in $v_{\phi}$ as functions of height for the nonmoving and moving frames are quite similar. (We do not show the rms profile for the uniform moving frame case because it is similar to that of the nonmoving retrieval due to the weak mean flow at this time.) The rms errors are consistent with vertical profiles of the observed and retrieved mean tangential winds (not shown) which indicate that the moving reference frame out performs the nonmoving frame in the lower and upper levels only (from 3.0 to $9.0 \mathrm{~km}$, the retrieved

moving and stationary frame $\bar{v}_{\phi}$ were close to zero). Midlevel rms errors for the two reference frames are quite similar. In contrast, in the regions where the mean flow is large, that is, near the surface and the domain top $(z>11 \mathrm{~km})$, the moving frame significantly improves the results. Below $2.5 \mathrm{~km}$, the rms errors for the moving frame retrieval are less than the stationary frame, with the largest improvement just above the surface where the moving frame rms is approximately 2.5 $\mathrm{m} \mathrm{s}^{-1}$ less than the stationary frame. The advantage of the moving frame above $9 \mathrm{~km}$ is also clearly demonstrated as it yields rms errors that are as much as 4.5 $\mathrm{m} \mathrm{s}^{-1}$ less than the stationary frame. The general increase in rms errors in these regions (i.e., above $9 \mathrm{~km}$ 

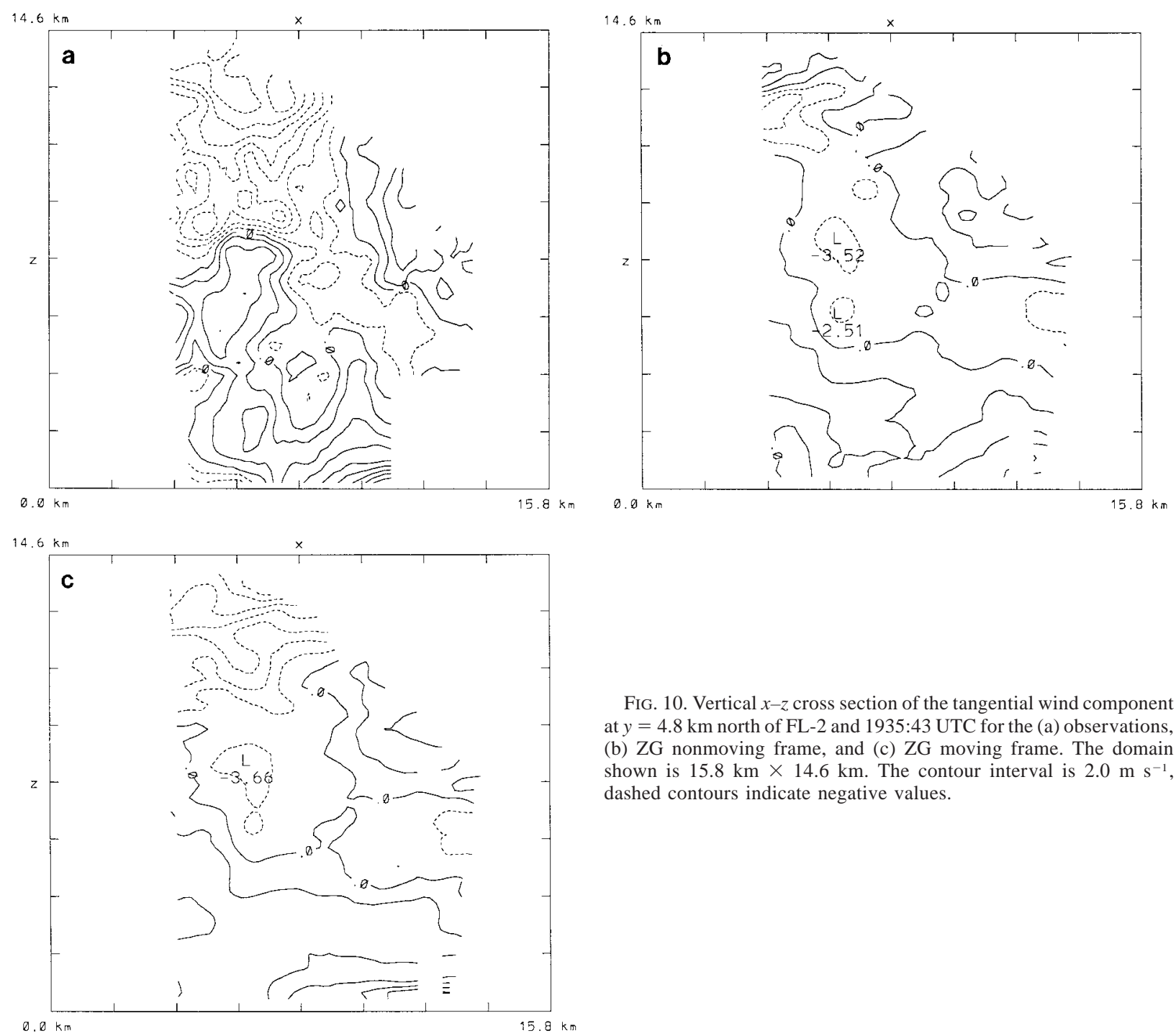

FIG. 10. Vertical $x-z$ cross section of the tangential wind component at $y=4.8 \mathrm{~km}$ north of FL-2 and 1935:43 UTC for the (a) observations, (b) ZG nonmoving frame, and (c) ZG moving frame. The domain shown is $15.8 \mathrm{~km} \times 14.6 \mathrm{~km}$. The contour interval is $2.0 \mathrm{~m} \mathrm{~s}^{-1}$, dashed contours indicate negative values.

and below $1.5 \mathrm{~km}$ ) for both reference frames primarily reflects the larger velocity magnitudes associated with the surface outflow and the upper-level divergence signatures. It is also possible that errors increase (above 9 $\mathrm{km})$ as a result of the large elevation angles. The combination of the high elevation angles and the proximity of the convection imply that the radial wind "sees" more of the Cartesian vertical component than it does the horizontal components. Consequently, it is possible that the retrieval has a difficult time gleaning information (about $U$ and $V$ ) from the radial wind constraint in the upper levels of the domain.

The weak constraint on the radial velocity given suggests that it might be possible to use the radial wind retrieval error as a possible gauge or quality control measure for the tangential component. Because radial wind measurements will be available, error statistics can be calculated and perhaps used to "flag" those regions, which have the potential to produce erroneous tangential wind information. Unfortunately, the results herein indicate no correlation between the errors in the retrieved radial component and those of the retrieved tangential component. To illustrate, Fig. 12 shows a vertical profile of the rms error in $v_{\phi}$ and $v_{r}$ for R35. The profile, representative of all three retrievals, indicates little, if any correlation between the errors in the radial and tangential winds. Attempts were made to evaluate the utility of other error measures, none of which indicated they could be used reliably as a quality control parameter.

\section{b. Comparison of R35, R26, and R29}

For comparison purposes we show the volume-averaged statistics for the retrieval presented in section $5 \mathrm{a}$ with those of R26 and R29 (Table 2). All variables have the same definitions as in Table 1. The results for all 


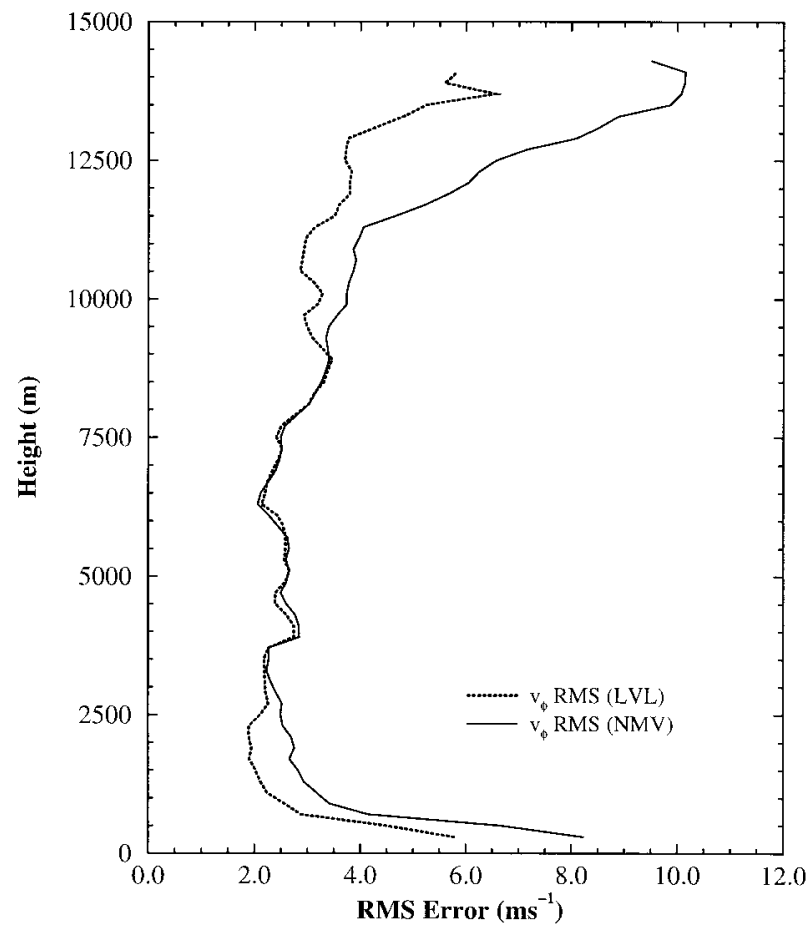

FIG. 11. The $z$ profile of the rms error in the tangential wind component for the nonmoving (solid curve) and moving (dashed curve) reference frames at 1935:43 UTC.

three retrievals are quite similar. R35 yields the best results with small tangential component errors and a volume correlation coefficient more than +0.20 better than for the other two retrievals. (The improved statistics may result from the storm complex entering a relatively steady period and/or a result of the closer proximity of the observations to the radar at this time.) Radial velocity statistics are calculated using all three retrieved components in Eq. (8), and calculated using Eq. (8) without the retrieved vertical velocity (denoted by an asterisk). The marked improvement reflected by the latter statistic suggests that the retrieved vertical velocity obtained from ZG has large errors (see section 6).

\section{Vertical velocity retrieval}

Heretofore the discussion has been limited to the retrieval of the tangential velocity component. At low elevation angles, the observed radial component and retrieved tangential component, in tandem, describe most of the observed horizontal flow (provided that the errors in the retrieved fields are not large). The results in section 5 indicate, for the most part, that retrieval error magnitudes (in the tangential component) are comparable to the discrepancy between the hybrid synthesis radial winds and those observed directly by the FL-2 radar (see Fig. 3). Heretofore, however, there has been no assessment of the retrieval of the vertical motion field. In this section we discuss the $\mathrm{ZG}$ retrieval of $w$

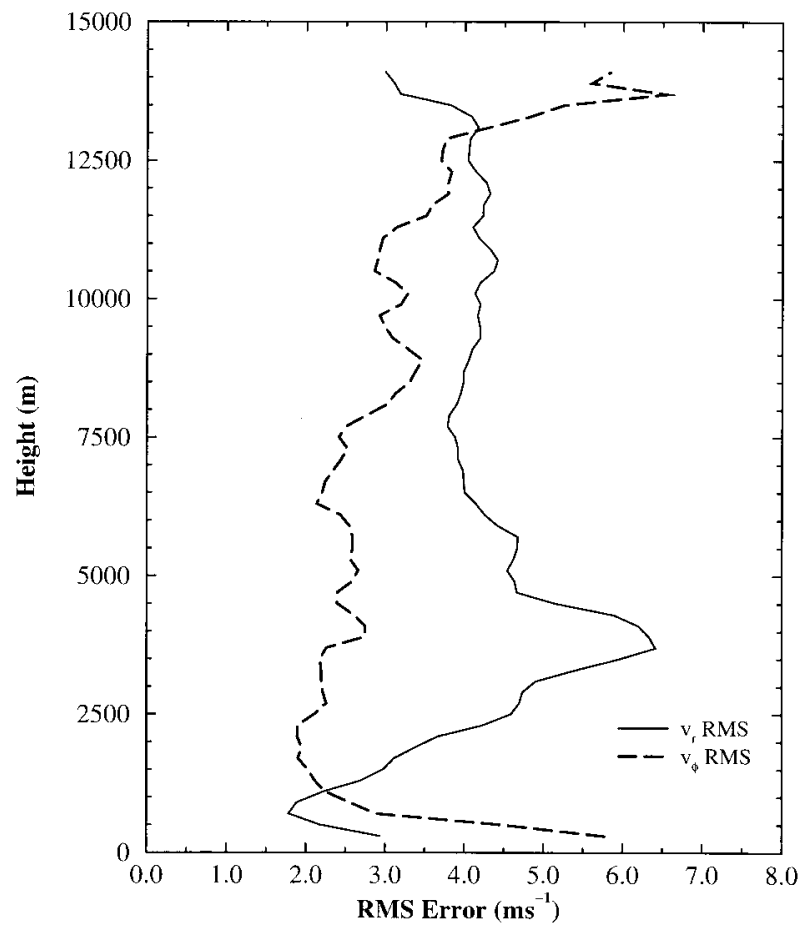

FIG. 12. The $z$ profile of the rms error in the retrieved tangential (dashed curve) and radial (solid curve) wind component at 1935:43 UTC for the moving frame.

for R35. The results for the other two retrievals are similar and will not be shown.

For brevity, we compare horizontal cross sections of the observed vertical velocity and that obtained from $\mathrm{R} 35$ at $z=5.1 \mathrm{~km}$ only (Figs. 13a,b, respectively). This level is representative of the overall performance of the $\mathrm{ZG} \mathrm{w}$ retrieval. The observations at $z=5.1 \mathrm{~km}$ indicate that a line of convection running southwest to northeast is recovered by $\mathrm{ZG}$, albeit somewhat crudely. The observations indicate sinking motion over the northern half of the domain, while the retrieval shows some sinking motion or relatively weak upward motion. At this level, $\rho=0.38$ and the rms error is relatively large $(5.7 \mathrm{~m}$ $\left.\mathrm{s}^{-1}\right)$. The volume-averaged $w$ rms error is $5.6 \mathrm{~m} \mathrm{~s}^{-1}$ (nearly twice that of the horizontal components) and $\rho$ is 0.27 .

The generally poor $\mathrm{w}$ retrievals obtained by the ZG scheme are likely a consequence of several factors, one of which involves the absence of a kinematic constraint on the vertical velocity (e.g., mass conservation; Liou 1999). In particular, this may explain the degraded results near the surface where the observed $w$ tends to zero but the retrieved $w$ can be arbitrary as it is not tied to any lower boundary condition. Errors associated with the terminal fall speed assumption may also be important. Another factor may be the moving frame definition itself-which takes into account the mean horizontal flow but not the vertical. (Results from our experiments suggest that $W$ is not zero.) Another potential influence 

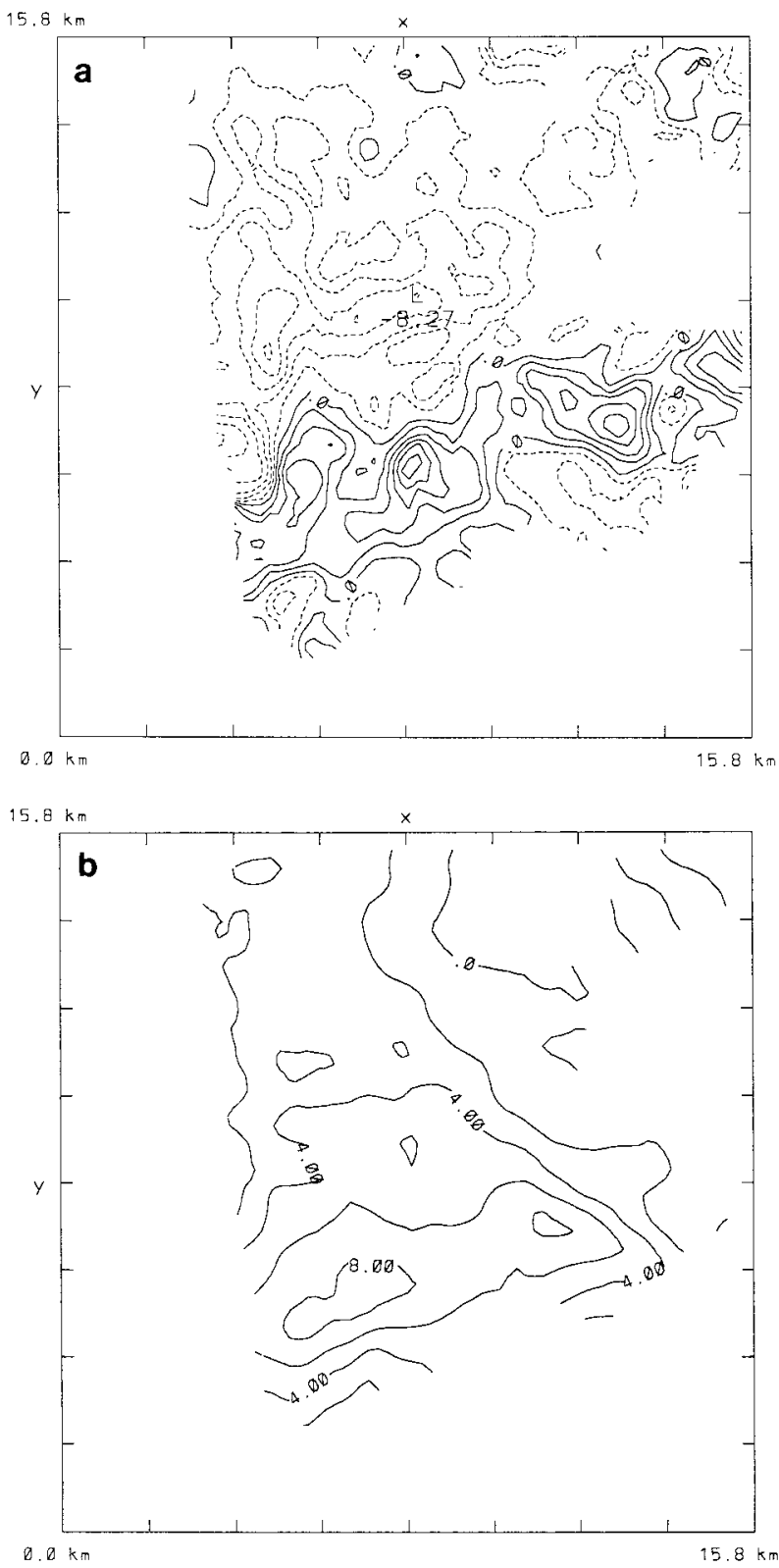

FIG. 13. Horizontal cross section of $w$ at $z=5.1 \mathrm{~km}$ and 1935:43 UTC for (a) observations, and (b) ZG-moving frame. The domain shown is $15.8 \mathrm{~km} \times 15.8 \mathrm{~km}$. The contour interval is $2.0 \mathrm{~m} \mathrm{~s}^{-1}$, dashed contours indicate negative values.

may be intrinsic to the flow itself whereby the surface outflow and upper-level divergence patterns are persistent features in contrast to the more transient nature of the updrafts over the 24-minute observation span. In the next section, we discuss several alternative methods to deduce $\mathrm{w}$ and compare these results with those obtained via the ZG methodology.

\section{a. Kinematic and O'Brien techniques}

Vertical velocities obtained using the retrieved horizontal wind field as input to the kinematic technique were unrealistic (not shown) - an artifact of the unspecified top boundary condition in combination with divergence estimates contaminated by error. This can be particularly problematic in the case where the horizontal wind field is provided by a retrieval. Because of the large errors in estimating $w$ using the kinematic technique, O'Brien (1970) sought an alternative method to obtain physically realistic values of the vertical motion field. By taking into account the variation of the divergence error with height, the variational formulation posed by O'Brien provides an objective adjustment to the divergence, which yields improved estimates of $w$. Our estimates of the vertical velocity using the O'Brien method were improved over that of the kinematic method. However, we still found the magnitude of the vertical velocity to be more than twice that of the observations. In contrast with the kinematic method, $w$ is fixed (set to zero) at both top and bottom boundaries in the O'Brien technique. This will likely improve the results near the boundary where the extra condition has been applied, provided that the observed $w$ actually approaches zero at that boundary.

\section{b. Modified O'Brien technique}

Because of the aforementioned difficulties associated with obtaining a realistic vertical velocity we derive an alternative method. Note that for the kinematic and O'Brien techniques discussed previously and the following technique, the vertical velocity is not estimated in the data-void regions. Estimates of the divergence on the periphery of the data region are also rejected. In addition, we use a variable elevation for the top of the integration domain-defined as the level (in that column) above which the reflectivity observations are missing.

The proximity of the convection to the radar suggests that the observed radial velocity, especially in the mid to upper levels of the storm, might be used to obtain an improved estimate of $w$. This, along with the results of the O'Brien technique, serves as the motivation behind the development of what we call the modified O'Brien method. In it, we use the divergence of the retrieved winds in the lower portion of the storm, along with the radial velocity in the mid-to-upper levels, so as to take advantage of the large correlation between the radial wind and the vertical velocity at high elevation angles.

Assuming that the radial velocity is observed and that the velocity retrieval yields estimates of the horizontal wind field, we can adjust the unknown vertical component subject to two weak constraints: mass conservation and a Cartesian coordinate-radial velocity relationship expressed by the following functional: 


$$
J=\int_{V}\left\{\alpha\left[\rho u^{r} x+\rho v^{r} y+\rho\left(w^{a}+W_{t}\right) z-\rho r v_{r}^{o}\right]^{2}+\left[\frac{\partial \rho u^{r}}{\partial x}+\frac{\partial \rho v^{r}}{\partial y}+\frac{\partial \rho w^{a}}{\partial z}\right]^{2}\right\} d V
$$

where $v_{r}^{o}$ denotes the observed radial velocity; $\alpha$ is a three-dimensional weight; $\rho$ is the base-state density (e.g., given by a sounding); $u^{r}$ and $v^{r}$ are the retrieved horizontal winds from $\mathrm{ZG} ; w^{a}$ is the adjusted (unknown) vertical velocity; $x, y$, and $z$ are the Cartesian coordinates; $r$ is the radar-to-grid point distance; $V$ represents a $3 \mathrm{D}$ volume in Cartesian space; $W_{t}$ is the raindrop terminal velocity defined by Eq. (2); and all other variables have their previously defined meanings. Bousquet and Chong (1998) combine elements of Eq. (8) above to perform a multiple-Doppler synthesis. Their analysis couples a least squares formulation of the overdetermined dual-Doppler problem (Ray and Sangren 1983) with mass conservation and smoothness constraints. Bousquet and Chong's technique, designed for multiple radars, simultaneously adjusts all three wind components (here we adjust the vertical component only). As an alternative to the modified O'Brien technique presented here, one could apply mass conservation directly to Eq. (2) — creating a modified ZG technique that directly builds in a dynamical constraint and adjusts all three wind components similar to the Bousquet and Chong. The advantage of the modified O'Brien (or ZG) technique over that of the Bousquet and Chong method is it requires only single-Doppler winds and reflectivity. Liou (1999) added a mass conservation constraint to the $\mathrm{ZG}$ method and produced improved retrievals of the horizontal wind components for a synthetic dataset at low-elevation angles. Analogous to the modified O'Brien technique presented here, Liou suggests that the vertical component can be derived from his modified $\mathrm{ZG}$ method if the horizontal components are retrieved with sufficient accuracy. Ideally, a combination of the Liou approach and that presented herein for the vertical velocity may produce the best $3 \mathrm{D}$ wind retrieval.

Anticipating that $\alpha$ should depend explicitly upon $z$, we set $\alpha(x, y, z)=\gamma(x, y) \times z^{p}$, where $p$ is an undetermined exponent. Minimizing Eq. (8) with respect to $\rho w^{a}$ and setting $J$ equal to zero yields a second-order inhomogeneous ordinary differential equation:

$$
\frac{d^{2} W}{d z^{2}}-\gamma W z^{2+p}=F
$$

where $F$ is given as

$$
\begin{aligned}
F= & \gamma z^{1+p}\left[\rho u^{r} x+\rho v^{r} y+\rho W_{t} z-\rho r v_{r}^{o}\right] \\
& -\frac{\partial}{\partial z}\left[\frac{\partial \rho u^{r}}{\partial x}+\frac{\partial \rho v^{r}}{\partial y}\right],
\end{aligned}
$$

$W=\rho w^{a}$, and as additional constraints, we require the vertical velocity to vanish at the domain top and bottom.
Applying the method of variation of parameters (e.g., Kreysig 1983), we can solve Eq. (9) and after some tedious algebra (see appendix for the details), arrive at the following solution:

$$
\begin{gathered}
W(z)=\sqrt{z} I_{\nu}(\eta)\left\{\frac{K_{\nu}[\eta(H)]}{I_{\nu}[\eta(H)]} \int_{0}^{H} 2 \sqrt{z^{*}} \nu F I_{\nu}(\eta) d z^{*}\right. \\
-\int_{0}^{H} 2 \sqrt{z^{*}} \nu F K_{\nu}(\eta) d z^{*} \\
\left.+\int_{0}^{z} 2 \sqrt{z^{*}} \nu F K_{\nu}(\eta) d z^{*}\right\} \\
-\sqrt{z} K_{\nu}(\eta) \int_{0}^{z} 2 \sqrt{z^{*}} \nu F I_{\nu}(\eta) d z^{*}
\end{gathered}
$$

where we have applied the impermeable boundary conditions on $W$. Here $I_{\nu}(\eta)$ and $K_{\nu}(\eta)$ are modified Bessel functions of the first and second kind, respectively, $\nu$ $=1 /(4+p), \eta=2 \nu \sqrt{\gamma} z^{1 / 2 \nu}, H$ is the domain depth, and the asterisk denotes a dummy variable.

The weights $(\gamma)$ are determined by the inverse variance of the integrated horizontal divergence and radial velocity in a vertical column; that is,

$$
\gamma(x, y)=\frac{\int_{0}^{H}\left(\frac{\partial \rho u^{r}}{\partial x}+\frac{\partial \rho v^{r}}{\partial y}\right)^{2} d z}{\int_{0}^{H}\left(\rho r v_{r}^{o}\right)^{2} z^{p} d z}
$$

We tested other weights, including a single volumeaveraged quantity (i.e., horizontally homogeneous), which yielded inferior results to those given by Eq. (12).

A series of experiments were conducted to determine the optimum value of $p$. Vertical velocity rms errors were calculated for a sequence of simulations using the $\mathrm{ZG}$ retrieved winds from the sensitivity experiment LVL given in Table 1. The lowest volume rms errors were found to occur for values of $p \approx 1.5$ (figure not shown). Thus, as the elevation angle increases, the relative importance of the radial velocity also increases. Intuitively, this makes sense because, as a scanning radar samples at higher elevation angles, it detects a larger fraction of the vertical velocity.

As in the kinematic and O'Brien techniques tested above, the input horizontal winds are those output from R26. Figure 16 depicts the retrieved vertical velocity field obtained via the modified O'Brien technique. The modified O'Brien technique yields magnitudes that are 


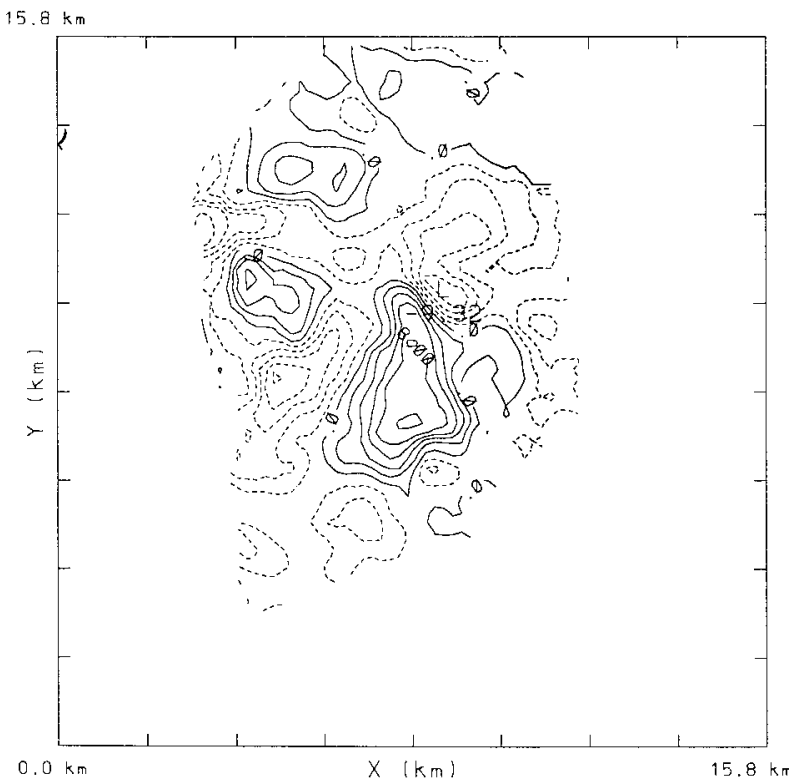

FIG. 14. Horizontal cross section of observed $w$ at $z=7.5 \mathrm{~km}$ and 1926:21 UTC. The domain shown is $15.8 \mathrm{~km} \times 15.8 \mathrm{~km}$. The maximum (minimum) observed $w$ is $10.4 \mathrm{~m} \mathrm{~s}^{-1}\left(-11.6 \mathrm{~m} \mathrm{~s}^{-1}\right)$. The contour interval is $2.0 \mathrm{~m} \mathrm{~s}^{-1}$, dashed contours indicate negative values.

much closer to that of the observed $w$ (Fig. 14) than the ZG method (Fig. 15). Significant improvements are otherwise obvious - especially the highly correlated updrafts and downdrafts. The rms errors as a function of height for the three techniques are shown in Fig. 17. The vertical error profile underscores the significant im-

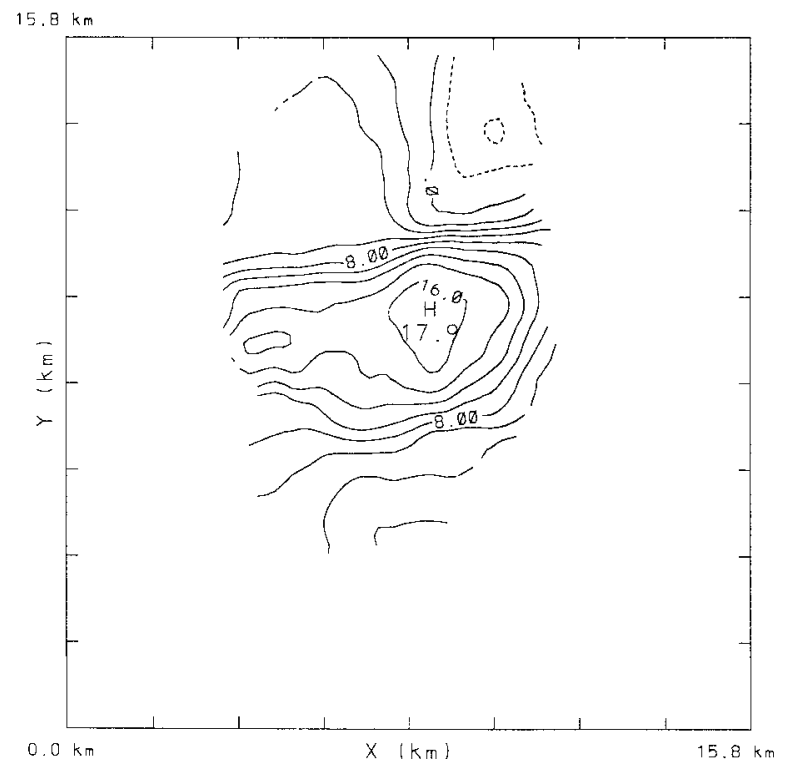

FIG. 15. Horizontal cross section of retrieved $w$ at $z=7.5 \mathrm{~km}$ and 1926:21 UTC for the ZG method. The domain shown is $15.8 \mathrm{~km} \times$ $15.8 \mathrm{~km}$. The maximum (minimum) $w$ is $17.9 \mathrm{~m} \mathrm{~s}^{-1}\left(-4.8 \mathrm{~m} \mathrm{~s}^{-1}\right)$. The contour interval is $2.0 \mathrm{~m} \mathrm{~s}^{-1}$, dashed contours indicate negative values.

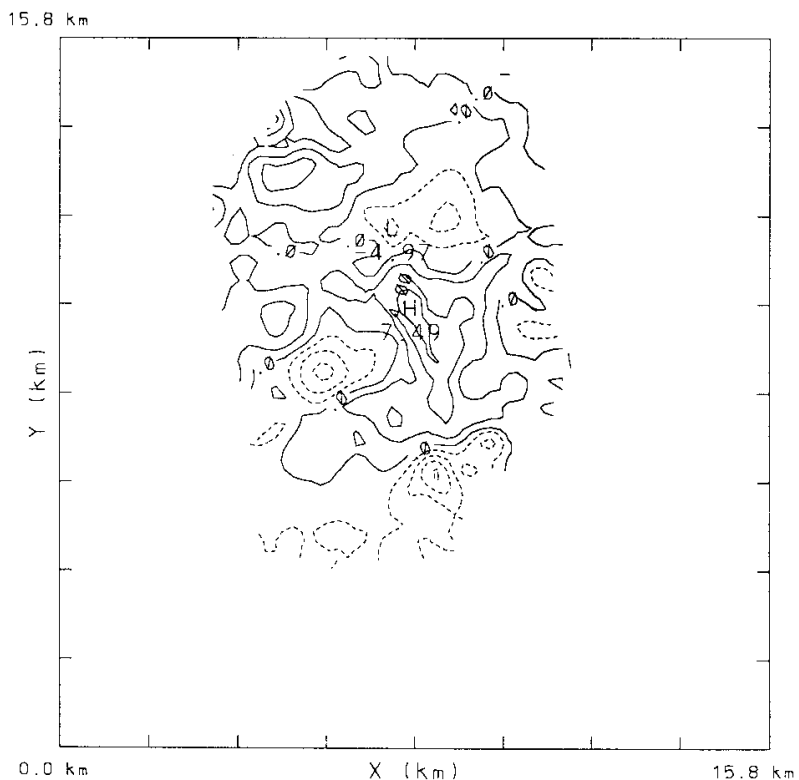

FIG. 16. Horizontal cross section of retrieved $w$ at $z=7.5 \mathrm{~km}$ and 1926:21 UTC for the modified O'Brien method. The domain shown is $15.8 \mathrm{~km} \times 15.8 \mathrm{~km}$. The maximum (minimum) $w$ is $24.3 \mathrm{~m} \mathrm{~s}^{-1}$ $\left(-18.9 \mathrm{~m} \mathrm{~s}^{-1}\right)$. The contour interval is $2.0 \mathrm{~m} \mathrm{~s}^{-1}$, dashed contours indicate negative values.

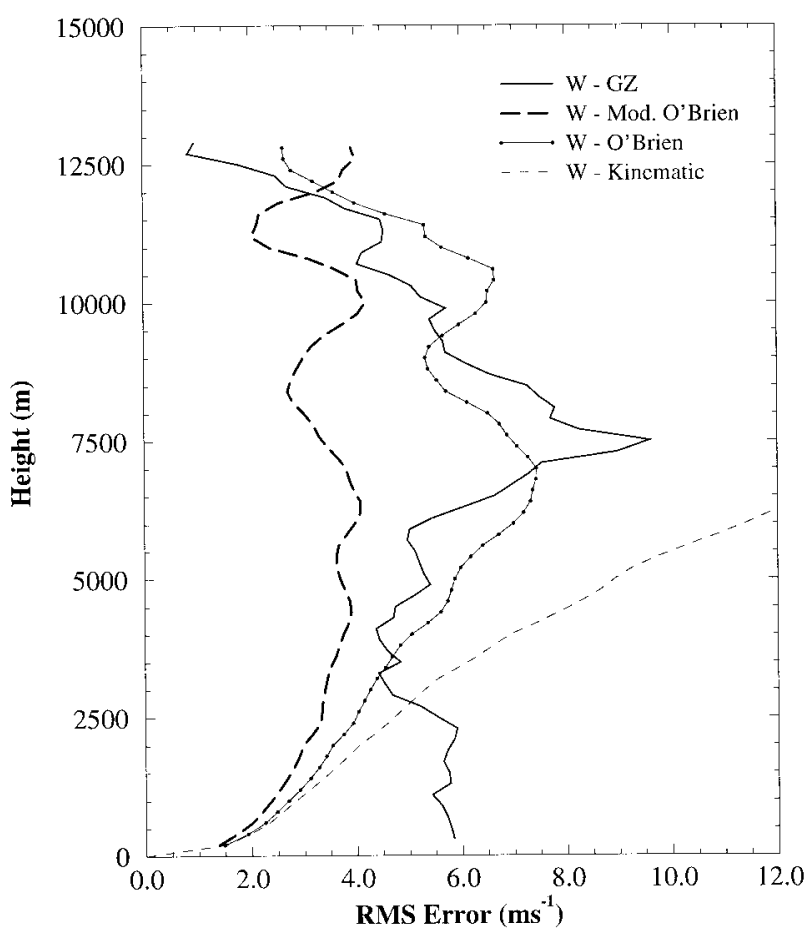

FIG. 17. The $z$ profile of the $w$ rms error at 1926:21 UTC for ZG (solid curve), modified O'Brien (thick dashed curve), O'Brien (solid curve with circles), and kinematic (thin dashed curve) techniques. 
TABLE 3. Vertical velocity rms errors. Units are $\mathrm{m} \mathrm{s}^{-1}$.

\begin{tabular}{lccc}
\hline \hline Technique/time & $165 \mathrm{~s}$ & $363 \mathrm{~s}$ & $727 \mathrm{~s}$ \\
\hline ZG & 5.87 & & \\
Kinematic & 12.1 & & \\
O'Brien & 5.42 & & \\
Mod O'Brien & 3.40 & 3.48 & 3.28 \\
\hline
\end{tabular}

provement achieved by applying the modified O'Brien scheme-especially at midlevels.

A summary of the volume rms error statistics is shown in Table 3. The results show a significant improvement in the rms errors for the modified O'Brien method-reflecting a combination of the removal of spurious updrafts/downdrafts, phase errors, and greater accuracy in the magnitude of $w$. Volume-averaged rms errors are reduced by nearly $50 \%$ over that of the O'Brien scheme and $100 \%$ over that given by the ZG retrieval. Comparison with Table 2 indicates that the vertical velocity errors for all three retrievals are comparable to the error levels for the tangential component.

\section{Discussion and summary}

We applied the Zhang-Gal-Chen (ZG) SDVR to retrieve the 3D wind field from the reflectivity and radial velocity observations of a single radar embedded in a triple-Doppler network. In an attempt to quantify our results, the retrieved winds were compared with the $3 \mathrm{D}$ wind field obtained via a synthesis of the radial wind from all three radars.

The application of ZG represents one of the first applications of a SDVR to deep convection. For the work presented herein, we use high-resolution observations of a transient multicell storm. The results of three experiments and a benchmark retrieval (each using the real data) indicate that the retrieval performs best when applied in a moving reference frame. Yet how one defines this moving frame is equally as important. Because SDVR has generally been limited to shallow phenomena such as a sea breeze or cold pool, the moving reference frame has been tied to the motion of somewhat isolated features that tend to move uniformly and in a single direction. Such is not the case for deep convection in a low-shear environment. Our results show that, in an environment with a hodograph that has critical layer, that is, where the wind components change sign at some height, the moving reference frame is best defined as a function of height rather than as some volume mean. The results also show that the benefit of the moving reference frame is best realized away from these critical layers, especially in regions where there is a substantial mean flow as in a surface outflow or the upper level divergence at storm top. While improving the results in these regions, the moving reference does not degrade the results in other more quiescent regions.

Though ZG was able to successfully retrieve the tangential component in many regions, it did not fair as well with the vertical wind component. Poor wind retrievals are likely to be problematic to the nowcaster and modeler-especially the latter as they will necessarily rely on SDVR to construct the initial conditions to simulate the evolution of a $3 \mathrm{D}$ atmosphere. As a result, concerted efforts were made to improve the vertical velocity obtained from $\mathrm{ZG}$, including the application of both the kinematic and O'Brien techniques. The horizontal winds obtained from ZG were used as input to each of these techniques but the results indicated rms errors comparable or larger than those of ZG did. The proximity of the radar to the storm, however, yields large correlations between the observed radial velocity component and hybrid synthesis vertical velocity-especially at mid-to-high elevations.

This suggested that, as a possible alternative, radial velocity information could be used to obtain an improved vertical motion field. This led to the development of what we refer to as a "modified O'Brien" technique. The scheme uses the observed radial velocity component and the retrieved horizontal wind field to deduce the convective-scale vertical velocity. Results indicate that this technique lowers the volume-averaged rms errors as much as $1.5-2.0 \mathrm{~m} \mathrm{~s}^{-1}$.

The advantage of this technique is that it utilizes only single-Doppler radar data and the retrieved horizontal winds to obtain an improved estimate of $w$ that approximately satisfies mass conservation and the observed radial wind. The method is pragmatic since it applies a relatively straightforward algorithm that incorporates data, which will generally be available. Additionally the scheme is efficient-using relatively little CPU time (on the order of a couple of minutes for both the ZG retrieval and $w$ adjustment). Thus, implementation as part of an assimilation scheme or its use for nowcasting remain viable options. Since the input to this technique requires the retrieved horizontal winds from $\mathrm{ZG}$, the single-Doppler reflectivity and radial velocity from at least two different volume scans are necessary. Once the ZG retrieval is performed, the retrieved horizontal winds, along with the observed radial velocity, can be fed into the modified O'Brien scheme to yield $w$ estimates, which are then used to replace those obtained via the ZG scheme. As a possible alternative, the ZG method could be reformulated to include the mass conservation constraint directly in Eq. (2)-circumventing the two-step process introduced herein. One potential advantage of modifying the ZG method directly would be that all three retrieved components would be affected. In a recent paper, Liou (1999) introduced a mass conservation constraint to the ZG method and produced improved retrievals of the horizontal wind components for a synthetic dataset at low-elevation angles. Liou suggests that the vertical component can be derived from his modified ZG method if the horizontal components are retrieved with sufficient accuracy. It is possible that a combination of the Liou approach, which yields improved horizontal wind com- 
ponents, and that presented herein for the vertical velocity may produce the best $3 \mathrm{D}$ wind retrieval. This does not diminish, however, our findings, which demonstrate that the choice of a particular reference frame can be important. Unlike some retrieval techniques, the ZG method can easily accommodate various reference frame definitions.

It is important to point out that the retrieved vertical motion field from the modified O'Brien technique may be overly optimistic due to the uncharacteristically large elevation angles and the storm proximity to the radar. Additionally, typical operational WSR-88D scan strategies produce volume scans on the order of $5 \mathrm{~min}-$ nearly twice that of the data used in this study. As shown here, even with high temporal resolution, rapidly evolving features may be problematic and the benefits of an increased number of volume scans to the retrieval may be somewhat reduced or offset by phase and amplitude errors in the retrieved wind field. Further evaluation of both the modified technique and the ZG methodology on other datasets is necessary, especially data obtained operationally where the observations are likely to be taken less frequently and at a greater distance from the radar. The question of what constitutes a "good" retrieval has been relegated to a somewhat simple evaluation in terms of rms error. While it would certainly be advantageous to use other indicators to gauge the quality of the retrieval, for example, a model initialization and forecast, we defer this to future work.

Acknowledgments. A special note of appreciation is in order for Dr. Tzvi Gal-Chen, who developed the particular SDVR technique investigated herein. Dr. GalChen passed away 5 years ago, yet the fact that his work continues to be of such utility reflects his intellectual pursuits and lasting contributions to the field of meteorology. We also extend a special thanks to our colleagues and friends, Sue Weygandt for assisting with the figures, and Jian Zhang, coauthor of the SDVR technique used herein. Our discussions stimulated many thoughts and ideas related to single-Doppler retrievals.

The hybrid wind synthesis dataset and soundings were obtained from Lincoln Laboratory with software support made available through Dr. Marilyn Wolfson of Lincoln Lab/MIT. The computations were performed on the IBM RISC System 6000 in conjunction with computer support from the Center for Analysis and Prediction of Storms. This research represents part of the lead author's Ph.D. thesis at the School of Meteorology, University of Oklahoma, and was supported by the National Science Foundation under Grants ATM91-20009 and ATM-92-22576.

\section{APPENDIX}

\section{Modified O'Brien Method to Determine the Storm-Scale Vertical Velocity}

If $F=0$ in Eq. (9), the complementary solution to the homogeneous problem is given by

$$
W_{c}(z)=\sqrt{z} L_{1 /(4+p)}\left(\frac{2}{4+p} \sqrt{\gamma} z^{(4+p) / 2}\right),
$$

where $L$ denotes a modified Bessel function (first or second kind) of real order $[1 /(4+p)]$. A general solution is given by a linear combination of the first and second modified Bessel functions, namely:

$$
W(z)=\sqrt{z} C_{1} I_{\nu}(\eta)+\sqrt{z} C_{2} e^{\nu \pi i} K_{\nu}(\eta)
$$

where $C_{1}$ and $C_{2}$ are coefficients to be determined, $I_{\nu}(\eta)$ and $K_{\nu}(\eta)$ are modified Bessel functions of the first and second kind, respectively, where:

$$
\nu=\frac{1}{4+p} \quad \text { and } \quad \eta=2 \nu \sqrt{\gamma} z^{1 / 2 \nu}
$$

Applying the method of variation of parameters (e.g., Kreysig 1983), we replace $C_{1}$ and $C_{2}$ above with variable coefficients, $A(z)$ and $B(z)$, and then solve for the particular solution. After some tedious algebra, we arrive at the following for $A$ and $B$ :

$$
\begin{aligned}
& A(z)=-\int_{0}^{z} \frac{F w_{2}\left(z^{*}\right)}{\mathrm{Wr}} d z^{*}+c_{1} \text { and } \\
& B(z)=\int_{0}^{z} \frac{F w_{1}\left(z^{*}\right)}{\mathrm{Wr}} d z^{*}+c_{2},
\end{aligned}
$$

where $F$ is given by Eq. (10) in the text, $w_{1}(z)=$ $\sqrt{z} I_{\nu}(\eta), w_{2}(z)=\sqrt{z} \mathrm{e}^{\nu \pi i} K_{\nu}(\eta)$, and Wr is the Wronskian given by

$$
\mathrm{Wr}=w_{1} w_{2}^{\prime}-w_{1}^{\prime} w_{2} .
$$

Here, the primes denote derivatives with respect to $z$.

Before arriving at the solution for $W$, we must determine the Wronskian in Eq. (A4) above. Differentiating $w_{1}$ and $w_{2}$ with respect to $z$ and using the recursion formula $L_{\nu}^{\prime}(\eta)=L_{\nu+1}(\eta)+(\nu / \eta) L_{\nu}(\eta)$ [where $L_{\nu}(\eta)$ denotes $I_{\nu}(\eta),\left(e^{\nu \pi i}\right) K_{\nu}(\eta)$, or any linear combination of these functions $]$ and the identity $\left[I_{\nu}(\eta) K_{\nu+1}(\eta)+\right.$ $\left.I_{\nu+1}(\eta) K_{\nu}(\eta)\right]=1 / \eta$ (Abramowitz and Stegun 1972), we arrive at the following:

$$
\mathrm{Wr}=-\frac{z}{\eta} \frac{d \eta}{d z} e^{\nu \pi i}=-\frac{e^{\nu \pi i}}{2 \nu} .
$$

Substituting (A6) and $d \eta / d z=\sqrt{\gamma} z^{(1-2 v) / 2 v}$ into Eq. (A4) we solve for the coefficients $A$ and $B$ and then substituting these into Eq. (A2) we arrive at the general solution:

$$
\begin{aligned}
W(z)= & \sqrt{z} c_{1} I_{\nu}(\eta)+\sqrt{z} c_{2} e^{\nu \pi i} K_{\nu}(\eta) \\
& +\sqrt{z} I_{\nu}(\eta) \int_{0}^{z} 2 \sqrt{z^{*}} \nu F K_{\nu}(\eta) d z^{*} \\
& -\sqrt{z} K_{\nu}(\eta) \int_{0}^{z} 2 \sqrt{z^{*}} \nu F I_{\nu}(\eta) d z^{*}
\end{aligned}
$$

We solve for the coefficients $c_{1}$ and $c_{2}$ by applying the 
impermeable boundary conditions on $W$. Note that $K_{v}$ grows without bound as $z$ approaches zero, and does so faster than $\sqrt{z} \rightarrow 0$ (Abramowitz and Stegun 1972); consequently, we set $c_{2}=0$. On the top boundary (at $z=H$ ) Eq. (A7) yields:

$$
\begin{gathered}
c_{1}=\frac{K_{\nu}[\eta(H)]}{I_{\nu}[\eta(H)]} \int_{0}^{H} 2 \sqrt{z^{*}} \nu F I_{\nu}(\eta) d z^{*} \\
-\int_{0}^{H} 2 \sqrt{z^{*}} \nu F K_{\nu}(\eta) d z^{*}
\end{gathered}
$$

where $\eta(H)=2 v \sqrt{\gamma} H^{1 / 2 v}$.

Substituting $c_{2}=0$ and Eq. (A8) back into Eq. (A7) yields the solution [Eq. (11) in the text] to the variational problem given by Eq. (8).

\section{REFERENCES}

Abromowitz, M., and I. E. Stegun, 1972: Handbook of Mathematics with Formulas, Graphs, and Mathematical Tables. National Bureau of Standards Applied Mathematics Series, Vol. 55, U.S. Dept. of Commerce, 1046 pp.

Armijo, L., 1969: A theory for the determination of wind and precipitation velocities with Doppler radars. J. Atmos. Sci., 26, 570573.

Bousquet, O., and Michel Chong, 1998: A Multiple-Doppler Synthesis and Continuity Adjustment Technique (MUSCAT) to recover wind components from Doppler radar measurements. $J$. Atmos. Oceanic Technol., 15, 343-359.

Carbone, R. E., M. J. Carpenter, and C. D. Burghart, 1985: Doppler radar sampling limitations in convective storms. J. Atmos. Oceanic Technol., 2, 357-361.

Crook, A., and J. D. Tuttle, 1994: Numerical simulations initialized with radar-derived winds. Part II: Forecasts of three gust front cases. Mon. Wea. Rev., 122, 1204-1217.

DeLaura, R., M. M. Wolfson, and P. S. Ray, 1989: A hybrid Cartesian windfield synthesis technique using a triple-Doppler radar network. Preprints, 25th Int. Conf. on Radar Meteorology, Paris, France, Amer. Meteor. Soc., 240-243.

Droegemeier, K. K., 1990: Toward a science of storm-scale prediction. Preprints, 16th Conf. on Severe Local Storms, Kananaskis Park, AB, Canada, Amer. Meteor. Soc., 256-262.

- 1997: The numerical prediction of thunderstorms: Challenges, potential benefits, and results from realtime operational tests. WMO Bull., 46, 324-336.

Gal-Chen, T., 1982: Errors in fixed and moving frame of references: Applications for conventional and Doppler radar analysis. J. Atmos. Sci., 39, 2279-2300.

Hane, C. E., 1993: Storm motion estimates derived from dynamicretrieval calculations. Mon. Wea. Rev., 121, 431-443.

Keohan, C. F., M. C. Liepins, C. A. Meuse, and M. M. Wolfson, 1992: Summary of triple-Doppler data; Orlando 1991. Federa Aviation Administration Project Report DOT/FAA/NR-92/2.
Kessler, E., 1969: On the Distribution and Continuity of Water Substance in Atmospheric Circulations. Meteor. Monogr., No. 32, Amer. Meteor. Soc., 84 pp.

Kreysig, E., 1983: Advanced Engineering Mathematics. 5th ed. John Wiley and Sons, $988 \mathrm{pp}$.

Lazarus, S. M., 1996: The assimilation and prediction of a Florida multicell storm using single-Doppler data. Ph.D. dissertation, University of Oklahoma, $345 \mathrm{pp}$. [Available from School of Meteorology, 100 East Boyd, Norman, OK 73019.]

- A. Shapiro, K. Droegemeier, 1999: Analysis of the Gal-ChenZhang single-Doppler velocity retrieval. J. Atmos. Oceanic Technol., 16, 5-18.

Lilly, D. K., 1990: Numerical prediction of thunderstorms-Has its time come? Quart. J. Roy. Meteor. Soc., 116, 779-798.

Liou, Y., 1999: Single radar recovery of cross-beam wind components using a modified moving frame of reference technique. J. Atmos. Oceanic Technol., 16, 1003-1016.

O'Brien, J. J., 1970: Alternative solutions to the classical vertical velocity problem. J. Appl. Meteor., 9, 197-203.

Ray, P. S., and K. L. Sangren, 1983: Multiple-Doppler radar network design. J. Climate Appl. Meteor., 22, 1444-1454.

, C. L. Zeigler, W. Bumgarner, and R. J. Serafin, 1980: Single and multiple-Doppler radar observations of tornadic storms. Mon. Wea. Rev., 108, 129-147.

Shapiro, A., S. Ellis, and J. Shaw, 1995: Single-Doppler velocity retrievals with Pheonix-II data: Clear air and microburst wind retrievals in the planetary boundary layer. J. Atmos. Sci., 52, $1265-1287$.

Sun, J., and A. Crook, 1994: Wind and thermodynamic retrieval from single-Doppler measurements of a gust front observed during Pheonix II. Mon. Wea. Rev., 122, 1075-1091.

_, and — 1998: Dynamical and microphysical retrieval from Doppler radar observations using a cloud model and its adjoint. Part II: Retrieval experiments of an observed Florida convective storm. J. Atmos. Sci., 55, 835-852.

Tuttle, J. D., and G. B. Foote, 1990: Determination of the boundary layer airflow from a single-Doppler radar. J. Atmos. Oceanic Technol., 7, 218-232.

Weygandt, S., A. Shapiro, and K. K. Droegemeier, 1995: Adaptation of a single-Doppler velocity retrieval on a deep-convective storm. Preprints, 27th Conf. on Radar Meteorology, Vail, CO, Amer. Meteor. Soc., 264-266.

Xu, Q., Chong-Jian Qiu, and Jin-Xiang Yu, 1994a: Adjoint method retrievals of low-altitude wind fields from single-Doppler reflectivity measured during Pheonix-II. J. Atmos. Oceanic Technol., 11, 275-288.

,-- , and $-1994 \mathrm{~b}$ : Adjoint method retrievals of low-altitude wind fields from single-Doppler wind data. J. Atmos. Oceanic Technol., 11, 579-585.

,$- \ldots$, H.-D. Gu, and Jin-Xiang Yu, 1995: Simple adjoint retrievals of microburst winds from single-Doppler radar data. Mon. Wea. Rev., 123, 1822-1833.

Yuter, S. E., and R. A. Houze 1995: Three-dimensional kinematic and microphysical evolution of Florida cumulonimbus. Part I: Spatial distribution of updrafts, downdrafts, and precipitation. Mon. Wea. Rev., 123, 1921-1940.

Zhang, J., and T. Gal-Chen, 1996: Single-Doppler wind retrieval in the moving frame of reference. J. Atmos. Sci., 53, 2609-2623. 